\title{
CONTRIBUTION A L'ÉTUDE DE L'UTILISATION DIGESTIVE DES PULPES DE BETTERAVES DESSÉCHÉES PAR LES PORCS ET LES RUMINANTS
}

\author{
PAR
}

\author{
A. M. LEROY, G. LERY, Z. ZELTER
}

Laboratoire de recherches de la Chaire de Zootechnie, Institut national agronomique, Paris

Pour une récolte annuelle moyenne de betteraves sucrières, la quantité totale de pulpe qui est mise à la disposition de l'Élevage français est de 7 millions de tonnes, ireprésentant à l'état frais 500.000 milliers d'unités fourragères, équivalant à 5 millions de quintaux de céréales. Cette pulpe est pauvre en matière sèche, et sa conservation dans le cours de l'hiver et au début du printemps pose des problèmes qui sont loin d'être tous résolus d'une manière satisfaisante. La pulpe doit être transportée de la sucrerie jusque dans les fermes, ce qui entraîne des déplacements longs et coûteux. La conservation en silos, entreprise trop souvent dans de très mauvaises conditions, s'accompagne de pertes qui peuvent atteindre à la fin de la période de conservation jusqu'à $60 \%$ des matériaux nutritifs initiaux.

Dans d'autres pays que le nôtre, et notamment en Allemagne, en GrandeBretagne et en Suède, ces pulpes sont séchées dans les usines, jusqu'à une teneur en matière sèche voisine de $90 \%$, ce qui permet de les conserver sans risquer de leur voir perdre leur valeur alimentaire, même après un stockage de plus d'une année. L'aliment ainsi obtenu se rapproche par sa composition des aliments concentrés, comme l'indique l'analyse suivante, qui provient des résultats moyens de 6 échantillons différents, examinés par la méthode de référence préconisée par 1'Institut Professionnel de Contrôle et de Recherches Scientifiques des Industries de l'Alimentation animale (I), (2), (3) sur des échantillons provenant de sucreries françaises outillées spécialement pour le séchage de leurs sous-produits.

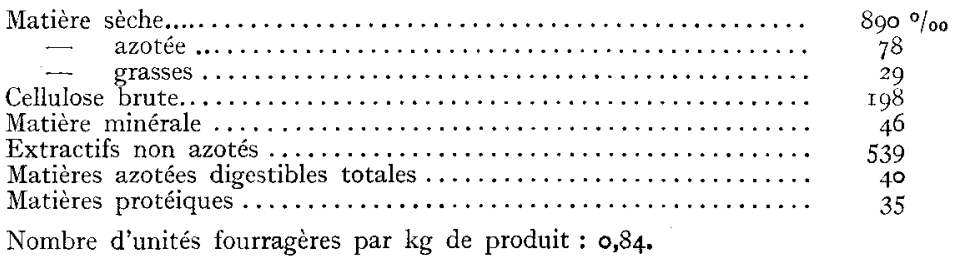


La teneur en matières azotées, bien que faible, n'est donc pas négligeable, et peut se comparer, à ce point de vue, à celles des caroubes dénoyautées.

Il existe, sur la valeur alimentaire des pulpes sèches, une importante documentation d'origine étrangère (4), (5), (6). - D'après une récente étude faite en Suède (7), la digestibilité de la matière organique contenue dans cet aliment serait, pour des vachęs en lactation, de $76,7 \%$, tandis que la digestibilité de la matière azotée correspondante ne serait que de $43,7 \%$.

Afin de renseigner nos éleveurs, nous avons cherché à vérifier l'exactitude de ces données, en travaillant successivement sur des bœufs, des moutons et des porcs. L'étude de la bibliographie nous avait d'ailleurs montré 1'abse nce presque complète de documents concernant 1'utilisation de la pulpe desséchée par les porcs.

\section{PRINCIPE ET CONDUITE DES EXPERIENCES}

Les animaux que nous avons mis en expérience ont été d'abord soumis à une alimentation témoin, dépourvue de pulpe. Dans une deuxième phase des essais, une partie de l'aliment de référence a été remplacée par de la pulpe. La comparaison, dans chaque cas, des ingesta et des excreta solides nous a permis de calculer par différence les coefficients de digestibilité des divers constituants de la pulpe.

Nous savons évidemment que cette méthode n'est pas à l'abri des critiques, en raison de l'influence bien connue de l'introduction d'un nouvel aliment sur la digestibilité de la fraction de la ration primitive, mais la répétition des essais avec, dans la mesure du possible, des animaux d'espèces différentes, nous a permis d'éviter en grande partie ces inconvénients.

Les périodes d'études proprement dites ont été en moyenne de Io jours, afin d'éliminer les 'erreurs dues aux irrégularités de l'émission des déchets digestifs. Les périodes d'adaptation aux différents régimes étudiés ont été plus ou moins longues, suivant les espèces et l'importance des variations du régime alimentaire ; nous avons fixé leur durée minimum à 6 jours pour les expériences sur porcs, et à ro jours pour les expériences avec ruminants.

Nous avons pu étudier chez les ovins la digestion de la pulpe sèche distribuée seule, au cours d'une période de 7 jours ; il ne nous a malheureusement pas été possible de prolonger plus longtemps cet intéressant essai, à cause de l'apparition d'une diarrhée sanguinolente observée sur 1'un de nos sujets, prélude de troubles plus graves.

\section{PROTOCOLE EXPERIMENTAL}

\section{Choix des animaux}

Les porcs et les brebis ont été choisis dans des élevages inscrits aux Livres Généalogiques de leur race, pratiquant depuis longtemps la sélection, de façon 
à pouvoir utiliser des animaux aussi homogènes que possible. Les bœufs nous ont été aimablement prêtés par des agriculteurs de la région parisienne, pour la durée de nos essais.

\section{Méthode de prélèvement des matières fécales}

Les porcs et les brebis ont été placés séparément dans des cages métalliques dont la description a été donnée précédemment par nous ; le protocole expérimental que nous avons utilisé a également fait l'objet d'explications détaillées(8).

Deux systèmes de collecte de matières fécales ont été utilisés pour les boufs, basés respectivement sur 1'emploi d'un harnais spécial ou sur 1'aménagement de stalles appropriées.

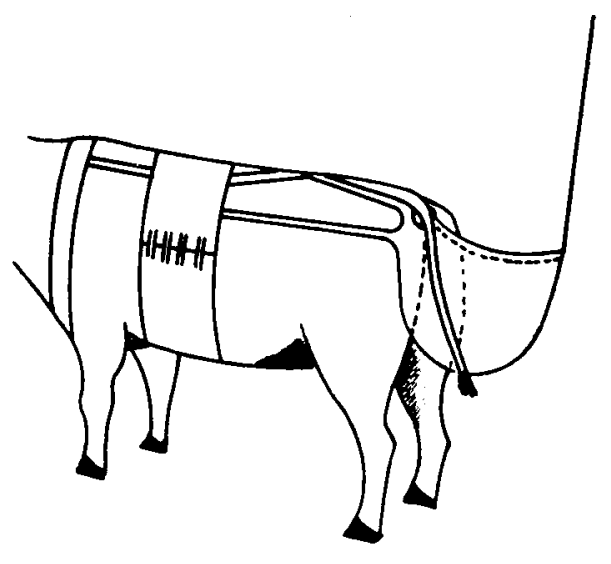

Schèma 1

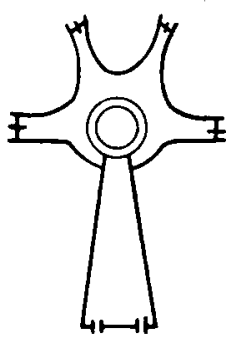

Schéma 2

Dans le système des harnais, on recueillait les matières fécales dans un sac de toile caoutchoutée résistante, portant une ouverture circulaire appliquée autour de l'anus de l'animal. Ce sac était retenu par deux courroies dorsales que l'on entrecroisait, complétées de deux courroies latérales prenant toutes les quatre appui sur tune large sangle entourant la poitrine de l'animal. Un léger collier relié à la sangle empêchait cette dernière de glisser en arrière. Une large bande de toile partait du bord inférieur de l'ouverture du sac et descendait entre les cuisses de l'animal. Des courroies ventrales reliaient cette bande à la sangle de poitrine. Le sac comportait à sa partie supérieure une large fente que l'on pouvait ouvrir pour procéder à la récolte des matières fécales, et refermer au moyen d'une fermeture-éclair. Un anneau permettait de soutenir la partie postérieure du sac à une tringle fixée au plafond afin d'éviter que le poids des matières fécales soit supporté entièrement par l'animal.

Les bœufs étaient placés dans une étable du type classique " tête au mur " 
que M. THIERY, Directeur du Laboratoire de recherches vétérinaires d'Alfort, avait aimablement mise à notre disposition. Ces stalles comportaient une auge et un râtelier ; les animaux étaient régulièrement abreuvés au seau.

Ce système présentait deux inconvénients. Il arrivait parfois que les animaux en remuant déplaçaient légèrement l'ouverture du sac par rapport à l'anus ; une partie des matières fécales tombait alors sur le sol ; ces matières étaient facilement récupérables, car les animaux n'avaient pas de litière.

D'autre part, le système de courroies et de sangles fatiguait les animaux. Le poids du harnais plein atteignait de 5 à $20 \mathrm{~kg}$; il fallait donc sangler très fortement les sujets pour que l'ensemble ne glisse pas vers l'arrière. L,es courroies frottaient toujours aux mêmes endroits du corps et les animaux en fin d'expérience, ont présenté des blessures de la peau assez gênantes et douloureuses.

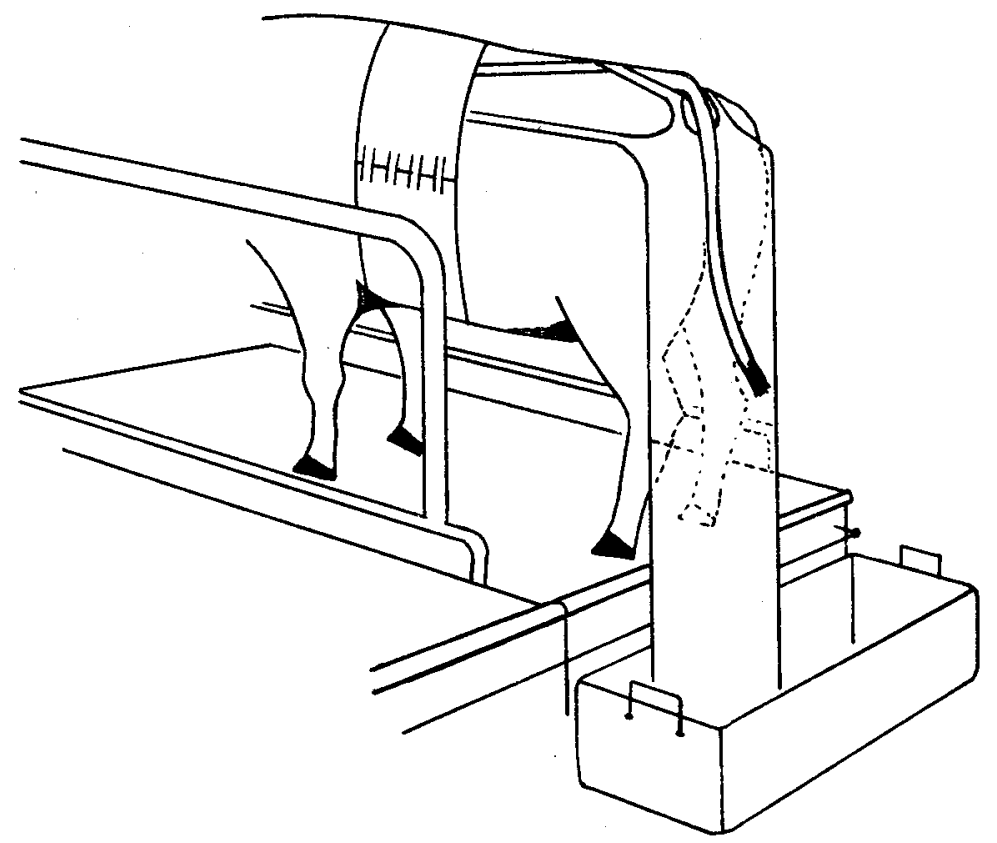

\section{Schéma 3}

Pour améliorer les conditions de vie de nos sujets, nous avons mis au point un système de stalles courtes, étroites et surélevées. Le schéma no 3 ciannexé montre comment a été conçue cette installation. Pour recueillir sans la moindre perte les matières fécales nous avons fixé à la partie postérieure de nos bœufs, au lieu d'un harnais, un grand tablier de caoutchouc qui conduisait les fèces directement dans un bac. Les sangles étaient nettement moins serrées que dans le système précédent puisqu'elles ne supportaient que le poids du tablier. 
Nous avions placé, de plus, pour protéger le garrot de l'animal du frottement de la sangle, un coussinet protecteur. Les boufs n'avaient pas de litière; les parties antérieure et postérieure de la stalle étaient recouvertes de briques creuses rainées (briques $Z \mathrm{E}, \mathrm{B}$ ). La partie centrale en ciment était légèrement creusée en forme de cuvette, afin de permettre la récolte de l'urine.

Un grand bouclier de tôle d'aluminium prévenait les projections horizontales de matières fécales, toujours possibles avec des régimes émollients, et permettait de les récupérer sans perte.

Une grille mobile autour de son axe inférieur était placée entre l'animal et son auge, et servait à régler à volonté la position du corps de l'animal. L'utilité de ce dispositif était de maintenir l'arrière-train de l'animal aussi près que possible du bord postérieur de la stalle. Un léger rebord arrondi empêchait l'animal de déraper et de glisser en arrière. Une barre métallique scellée dans la paroi prévenait par sa saillie la chute accidentelle de l'animal dans le bac de récolte. Les sujets étaient attachés par l'encolure au moyen d'un collier métallique du type américain, et la largeur des stalles avait été calculée de manière à limiter le plus possible les mouvements latéraux de nos animaux, conditions indispensables à la récolte convenable des fèces.

Ajoutons, enfin, que des abreuvoirs automatiques leur permettaient de boire à volonté.

Après une période d'adaptation, nous avons reconnu la nécessité de faire circuler chaque jour les animaux pendant un quart d'heure au moins, afin de leur éviter l'ankylose par un léger exercice. Un dressage rapide leur a permis de quitter leur stalle et de la rejoindre sans difficulté, à l'aide d'un plan incliné mobile spécialement aménagé à cet effet. Une récupération aussi complète que possible des matières fécales émises exceptionnellement en dehors des stalles a permis d'éviter les pertes qui auraient au se produire dans cette éventualité.

\section{La distribution des repas}

Les pores recevaient deux repas par jour, le matin vers ro heures, après le nettoyage des cages, et le soir vers 18 heures. Les deux repas étaient identiques et toujours présentés sous forme d'une bouillie liquide.

Les repas des brebis étaient composés de foin et de pulpe. Pendant les essais avec pulpe, cet aliment était distribué le matin, ce qui permettait de surveiller les sujets et d'éliminer les refus pour les peser. Le foin était donné en une ou deux fois, selon l'importance de la ration consommée, qui variait avec le taux de pulpe dans la ration. La plus forte ration de foin ou la ration unique de ce fourrage était distribuée le soir, de manière à permettre aux bêtes de manger à leur guise pendant la nuit. Les refus étaient ainsi recueillis et pesés le matin.

Pour éviter le gaspillage et récupérer plus facilement les refus, nous avons coupé le foin au hachoir, de manière à obtenir des tronçons de 3 à $4 \mathrm{~cm}$ de 
longueur. Cette façon de procéder nous a permis une récolte complète, ainsi qu'un bon échantillonnage des refus.

Le rythme de distribution était le même pour les bœufs que pour les brebis. La seule différence provenait de ce que le foin destiné aux bœufs était distuibué sans hachage préalable.

\section{Les échantillons}

Pour tous les animaux, les récoltes étaient effectuées tous les matins. Les matières fécales et 1'urine étaient échantillonnées, proportionnellement, pour les premières à leur poids, et pour la seconde, à son volume. Les échantillons de matières fécales étaient immédiatement séchés jusqu'à poids constant à l'étuve à $100^{\circ}$ et à partir d'octobre I949, avec l'aide d'un séchoir à rayons infra-rouges. Après séchage, elles étaient conservées et formaient ainsi en fin d'expérience un échantillon global moyen destiné à l'analyse. L'urine était mise en glacière avec quelques gouttes de chloroforme jusqu'au moment de son examen au laboratoire.

Sur tous les aliments distribués, on effectuait journellement un prélèvement pour le dosage de la matière sèche et les échantillons ainsi prélevés taient réunis en un échantillon moyen soumis à l'analyse complète.

En faisant usage de leurs abreuvoirs automatiques, les bœufs mouillaient leurs refus de foin, ce qui nous a obligés à effectuer journellement les dosages de la matière sèche sur lesdits refus ; d'autre part, nos animaux triaient leur foin pour en consommer les parties les plus fines, ce qui nous a obligés à effectuer une analyse spéciale de ces refus, afin de tenir compte des particularités de leur composition dans nos calculs. Toutes les analyses ont été effectuées par les méthodes précédemment indiquées (I), (2), (3), sous la direction de A. Françors, Chargé de Recherches.

\section{EXPERIENCES SUR PORCS}

Trois porcs Large White, âgés de 3 mois $\mathrm{I} / 2$ au début de l'expérience, ont été observés depuis octobre I945 jusqu'à janvier I946.

En raison de la teneur en matières cellulosiques de la pulpe, qui est plus élevée que celle des aliments ordinaires du porc, nous avons été amenés à concevoir un régime de comparaison de richesse cellulosique identique à celui qui comportait des pulpes, en incorporant à un aliment de base formé d'aliments concentrés (céréales, tourteaux, farine de viande) de la paille de blé finement broyée.

Nous avons tenté d'incorporer dans le régime expérimental une quantité de pulpe sèche aussi élevée que possible. Après avoir constaté des accidents de diarrhée pour des teneurs supérieures à $20 \%$, nous avons maintenu ce pour- 
centage à $16,8 \%$ (1) pendant les deux périodes expérimentales. Au cours de la première période, la pulpe a été utilisée après un broyage simple, tandis que pour la seconde période, nous avons poussé le broyage jusqu'à éclatement des parois cellulaires $\left({ }^{2}\right)$. Tous les animaux ont été soumis ensemble et successivement aux différents régimes, distribués dans l'ordre ci-après : régime témoin, régime avec pulpe broyée $E_{1}$, régime avec pulpe éclatée $E_{2}$.

La croissance a été régulière et constante au cours des essais. Le gain moyen de poids vif a été de $450 \mathrm{~g}$ par jour pour les porcs $\mathrm{n}^{0} \mathbf{I}$ et $\mathrm{n}^{\circ} 2$, et de $580 \mathrm{~g}$, pour le porc $\mathrm{n}^{\circ} 3$.

Le tableau I bis, ci-après donne les résultats d'analyses des aliments utilisés au cours des expériences, ainsi que celles des fèces émises pendant les périodes correspondantes.

\section{TABLEAU I}

\begin{tabular}{|c|c|c|c|c|c|c|c|}
\hline & $\begin{array}{l}\text { Matière } \\
\text { sèche }\end{array}$ & $\begin{array}{l}\text { Mat. } \\
\text { miné- } \\
\text { rale } \\
-\end{array}$ & $\begin{array}{l}\text { Mat. } \\
\text { orga- } \\
\text { nique }\end{array}$ & Azote & $\begin{array}{l}\text { Mat. } \\
\text { grasse }\end{array}$ & $\begin{array}{l}\text { Cellu- } \\
\text { lose } \\
\end{array}$ & $\begin{array}{c}\text { Extractifs } \\
\text { non } \\
\text { azotés } \\
-\end{array}$ \\
\hline Aliment témoin $\mathrm{T}$. & I 000 & 66,9 & $933, \mathrm{I}$ & 29,7 & $4 I, 0$ & 143,5 & 570,6 \\
\hline Fèces porc no $n^{\circ} \ldots \ldots \ldots \ldots \ldots \ldots$ & I 000 & 108,6 & 891,4 & I9, I & 38,3 & 344,0 & $395, \mathrm{I}$ \\
\hline$-n^{0} 2 \ldots \ldots \ldots \ldots \ldots \ldots \ldots$ & I 000 & 97,0 & 903,0 & $x, 1$ & 36,2 & 342,2 & 410,4 \\
\hline$--n^{\circ} 3 \ldots \ldots \ldots \ldots \ldots \ldots$ & I 000 & $10,5,2$ & 894,8 & r9,6 & 38,0 & 316,7 & 423,0 \\
\hline 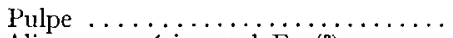 & I 000 & 60,7 & 939,3 & 24,7 & 30,9 & 217,4 & $54^{2,6}$ \\
\hline Aliment expérimental $\mathrm{E}_{1}\left(^{(3}\right)$ & I 000 & 60,7 & 939,3 & 27,6 & 42,6 & 177,8 & 553,4 \\
\hline 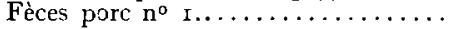 & 1000 & 104,6 & 895,4 & 20,1 & 39,9 & $3 \mathrm{I} 7,2$ & 417,7 \\
\hline$-r-n^{0} 2 \ldots \ldots \ldots \ldots \ldots \ldots$ & I 000 & 89,3 & 910,7 & I 9,8 & 34,0 & $357, \mathrm{I}$ & $40 \mathrm{I}, \mathrm{I}$ \\
\hline$-\mathrm{n}^{\mathrm{o}} 3 \ldots \ldots \ldots \ldots \ldots \ldots \ldots$ & I 000 & 104,8 & 893,9 & 22,9 & $46, \mathrm{I}$ & 3 I $5, \mathrm{I}$ & $395, \mathrm{I}$ \\
\hline Aliment expérimental $\mathrm{E}_{2}\left({ }^{3}\right) \ldots$ & I 000 & 65,4 & 934,5 & 28,0 & $4 \mathrm{I}, 8$ & I 75,4 & 549.2 \\
\hline Fèces porc $n^{0} x \ldots \ldots \ldots \ldots \ldots \ldots$ & I 000 & 107,6 & 892,4 & 22,0 & 38,3 & $3 \mathrm{I} 9,5$ & 402,7 \\
\hline$-1-n^{\circ} 2 \ldots \ldots \ldots \ldots \ldots \ldots$ & I $\infty 00$ & 99,2 & 900,8 & 22,4 & 40,5 & 319,5 & 406,2 \\
\hline$--n^{\circ} 3 \ldots \ldots \ldots \ldots \ldots \ldots$ & I 000 & 104,8 & 895,2 & 23,6 & 37,4 & 318,0 & 398,0 \\
\hline Eau de lavage moyenne $\%$ & $7, \mathrm{I}$ & $\mathrm{I}, \mathrm{I}$ & 6,0 & 0,4 & & & \\
\hline
\end{tabular}

Nous avons été amenés à apporter une correction aux quantités de matières fécales recueillies afin de tenir compte des éléments les plus fins de ces matières entraînés avec les eaux de lavage. Pour effectuer cette correction, le volume quotidien de ces eaux a cété ajusté à 6 litres.

La teneur en matières sèches des matières fécales récoltées a été de $20 \%$. Elle s'est maintenue relativement constante pour tous les animaux et pour tous les régimes.

Le tableau II donne les coefficients de digestibilité des matériaux ingérés pour les différents régimes. Nous publions en annexe à la fin du rapport les détails des calculs qui ont permis d'arriver à ces résultats.

Les erreurs-types permettent de s'assurer de la précision des principaux résultats.

(1) Ce pourcentage correspond à l'expression $\frac{\text { matière sèche de la pulpe ingérée }}{\text { matière sèche totale ingérée }} \times$ roo.

${ }^{2}$ ) Cet éclatement a été obtenu par hydratation du produit et passage entre des rouleaux compresseurs et sécheurs (procédé Doitteau).

$\left.{ }^{3}\right)$ Les aliments $E_{1}$ et $E_{2}$ contiennent $16,8 \%$ de pulpe. 


\section{Tableau II}

Coefficients moyens de digestibilité pour les différents régimes expérience.

\begin{tabular}{|c|c|c|c|c|}
\hline Eléments & $\begin{array}{l}\text { Régime témoin } \\
\text { (moyenne de } 3 \\
\text { résultats) }\end{array}$ & $\begin{array}{l}\text { Régime mixte } \\
\text { (moyenne de } 3 \\
\text { résultats) }\end{array}$ & $\begin{array}{r}\text { Pulpe (ca } \\
\text { par différ } \\
\text { (moyenne de } 6\end{array}$ & $\begin{array}{l}\text { alculée } \\
\text { rence) } \\
6 \text { résultats) }\end{array}$ \\
\hline Matière sèche. & 53,9 工二 $0,3(1)$ & 60,7 亡 0,6 & $92,7 \bar{I}$ & $3, I$ \\
\hline - minérale.............. & $26,6=2,1$ & $35,0 \mp 1,4$ & $74,9 \mp$ & 7,2 \\
\hline - organique............ & $55,9-0,4$ & $62,4 \mp 0,65$ & $93,5 \div$ & 3,2 \\
\hline Azote $\ldots \ldots \ldots \ldots \ldots \ldots \ldots$ & $67,3+0,4$ & $67,2 \div 0,8$ & $66,8 \mp$ & 3,9 \\
\hline Matière grasse. . & $59,6 \div \mathbf{I}$ & $64,3 \mp 1,6$ & 86,5 工 & 9,0 \\
\hline - cellulosique $\ldots \ldots \ldots \ldots$ & 0,2 & $29,7 \mp 9,1$ & $(\mathbf{r} 69,9) \mp$ & 12,0 \\
\hline Extractif non azoté $\ldots \ldots \ldots \ldots$ & $68,0 \quad 0,8$ & $72,1 \mp 0,4$ & $9 \mathbf{I}, \mathbf{I} \mp$ & 3,6 \\
\hline
\end{tabular}

En raison des faibles quantités de cellulose ingérée et de l'imperfection relative de la méthode empirique de dosage (méthode WEENDE) utilisée pour cet élément, les résultats correspondants sont évidemment entachés d'erreurs et l'application à ce cas particulier de la méthode par différence fait apparaître pour la cellulose de la pulpe une digestibilité supérieure à Ioo \% .

Il est d'ailleurs vraisemblable que la faible digestibilité de la cellulose observée au cours de l'expérience témoin correspondait à une insuffisante adaptation de l'appareil digestif de nos animaux à l'utilisation d'une dose anormalement élevée de cellulose. Notons, cependant que les régimes contenant de la pulpe de betterave étaient légèrement plus chargés de cellulose que le régime témoin : nous sommes amenés ainsi à conclure à l'amélioration de l'attaque des matières cellulosiques de la ration au cours de nos périodes expérimentales.

Le calcul de la valeur fourragère de la pulpe donne d'après la méthode mise au point par l'un de nous (9) avec les coefficients de digestibilité ci-après :

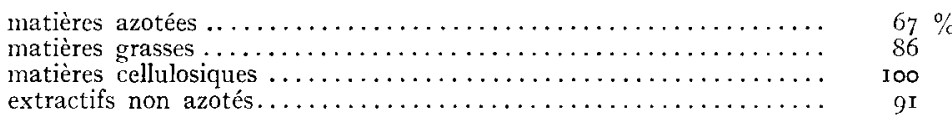

les valeurs suivantes :

a) pour la pulpe utilisée au cours de cette expérience (considérée à $890 \%$ de matière sèche) valeur fourragère par $\mathrm{kg}=\mathbf{I}, 03 \mathbf{u}$. $\mathrm{f}$.

b) pour la pulpe de composition moyenne citée aut début de ce mémoire valeur fourragère par $\mathrm{kg}=\mathrm{I}, 07 \mathrm{u} . \mathrm{f}$.

La pulpe sèche de betteraves s'est donc révélée comme un excellent aliment pour le porc, à la condition de ne pas être introduite dans les rations à des taux supérieurs à I5 \%. En raison de sa teneur en cellulose, cette pulpe doit être considérée comme un excellent aliment de lest, à la condition expresse de la faire tremper avant de la distribuer, afin d'éviter des accidents d'indigestion par surcharge. 


\section{EXPERIENCES SUR BOVINS ET OVINS}

Il nous a semblé plus logique, après examen des données, de présenter ensemble tous les résultats que nous avons obtenus en expérimentant avec des ruminants.

De mars à juin I946, nous avons étudié sur 3 brebis, âgées de 2 et 3 ans, de race Ile-de-France, la digestibilité d'un régime mixte de foin de luzerne et de pulpe, dans lequel le pourcentage de pulpe atteignait $46,1 \%$ (rapport de la matière sèche de la pulpe ingérée à la matière sèche totale ingérée), et nous l'avons comparée à celle [d'un régime de foin pur (groupe d'expériences $\left.\mathrm{n}^{\mathrm{o}} 2\right)$.

Ces expériences ont été reprises de février à mai I947, avec 3 autres brebis, de même race, âgées de 3 ans. Dans le régime mixte, le pourcentage de pulpe a été abaissé à 33,6\% (groupe d'expériences $\mathrm{n}^{\circ} 3$ ).

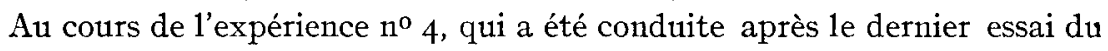
groupe 3 , les brebis ont reçu un régime composé exclusivement de pulpe.

Les premières expériences sur bovins ont été effectuées à 1'Ecole Vétérinaire d'Alfort, de décembre I947 à mai I948 (groupe d'expériences nº 5). Quatre bœufs de race normande, âgés de I 8 mois, ont reçu d'abord du foin seul, puis un mélange du même foin et de pulpe. Nous avons cherché à incorporer dans l'alimentation de ces animaux la plus forte quantité possible de pulpe. Après avoir constaté des accidents digestifs pour des pourcentages supérieurs à $50 \%$, nous avons fixé le taux de pulpe dans le régime expérimental à $42 \%$.

Puis, de novembre I949 à mars I950, nous avons effectué à l'Institut National Agronomique la série d'expériences du groupe 6, sur 2 bœufs de race Maine-Anjou, âgés de 20 à 24 mois. Le régime mixte expérimenté ne contenait que $29,5 \%$ de pulpe.

Tous les animaux ont maintenu leur poids sans gain ni perte, pendant la durée de ces expériences, sauf pendant l'expérience $n^{\circ} 4$, au cours de laquelle les brebis consommaient exclusivement de la pulpe en quantités simplement limitées par leur propre appétit.

Le tableau III fait connaître les résultats des analyses des foins, des refus de foin, des pulpes et des fèces correspondant à ces diverses expériences. Celles-ci ayant été effectuées à des époques différentes, nous avons utilisé pour chacune d'elles des foins et des pulpes de diverses origines, ce qui explique la variabilité des données de ce tableau.

Pour toutes ces expériences, les précautions prises pour la récolte des fèces et leur consistance nous a permis de les recueillir sans pertes.

Les teneurs en matières sèches des matières fécales des brebis ont montré des variations assez considérables avec le régime et avec l'animal étudié, et parfois au cours d'un même régime, d'un jour à l'autre. 


\section{TABLEAU III}

Composition des divers aliments et des fecès des expériences sur ruminants

\begin{tabular}{|c|c|c|c|c|c|c|c|}
\hline & $\begin{array}{l}\text { Matière } \\
\text { sèche } \\
-\end{array}$ & $\begin{array}{l}\text { Mat. } \\
\text { miné- } \\
\text { rale } \\
-\end{array}$ & $\begin{array}{l}\text { Mat. } \\
\text { orga- } \\
\text { nique } \\
\underline{-}\end{array}$ & $\begin{array}{c}\text { Azote } \\
-\end{array}$ & $\begin{array}{l}\text { Mat. } \\
\text { grasse } \\
-\end{array}$ & $\begin{array}{l}\text { Cellu- } \\
\text { lose } \\
-\end{array}$ & $\begin{array}{c}\text { Extracti } \\
\text { non } \\
\text { azotés } \\
-\end{array}$ \\
\hline oupe d'expériences no 2 : & & \multicolumn{6}{|c|}{$\begin{array}{c}\text { Brebis - } 3 \text { sujets } \\
\text { Période témoin }\end{array}$} \\
\hline & I 000 & $80, \mathrm{I}$ & 919,9 & 29,8 & $3^{2,0}$ & 308,1 & 406,4 \\
\hline & 1000 & 52,9 & 947,0 & $x 8,6$ & 45 , I & 439,6 & 350,6 \\
\hline rebis i $\ldots \ldots \ldots \ldots \ldots \ldots$ & I 000 & I 22,9 & $877, I$ & 18,7 & $4 \mathrm{I}, 7$ & 425,2 & $298, \mathrm{I}$ \\
\hline$-12 \ldots \ldots \ldots \ldots \ldots \ldots$ & I 000 & 105,7 & 894,3 & 20,7 & 39,5 & $35^{8,3}$ & 372,5 \\
\hline$-\quad 13 \ldots \ldots \ldots \ldots \ldots \ldots$ & I 000 & I 20,4 & 879,6 & $2 \mathrm{I}, 5$ & $44, \mathrm{I}$ & 330,1 & 376,3 \\
\hline
\end{tabular}

Foin $\left.{ }^{1}\right) \ldots \ldots \ldots \ldots \ldots \ldots \ldots \ldots \ldots$ I 000

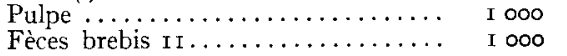

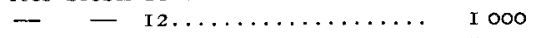

$-\quad-13 \ldots \ldots \ldots \ldots \ldots \ldots$ I 000

Groupe d'expériences no 3 :

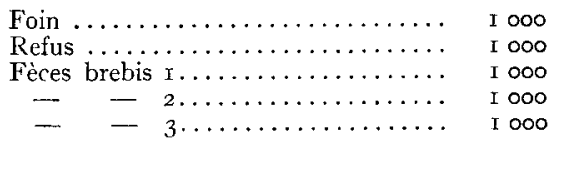

Foin $\ldots \ldots \ldots \ldots \ldots \ldots \ldots \ldots \ldots$ I 000

Refus $\ldots \ldots \ldots \ldots \ldots \ldots \ldots \ldots \ldots \ldots \ldots \ldots \ldots \ldots \ldots$ I 000

Pulpe .................. I 000

Fèces brebis $1 \ldots \ldots \ldots \ldots \ldots \ldots \ldots$ I 000

- $\quad-2 \ldots \ldots \ldots \ldots \ldots \ldots \ldots$ I 000

$-\quad-3 \ldots \ldots \ldots \ldots \ldots \ldots \ldots$ I 000

Groupe d'expériences no 4 :

Pulpe $\ldots \ldots \ldots \ldots \ldots \ldots \ldots \ldots \ldots$ I 1000

Fèces brebis $1 \ldots \ldots \ldots \ldots \ldots \ldots \ldots$ I 000

- $\quad 2 \ldots \ldots \ldots \ldots \ldots \ldots \ldots$ I 000

$-\quad-3 \ldots \ldots \ldots \ldots \ldots \ldots$ I 000

Groupe d'expériences $n^{\circ} 5$ :

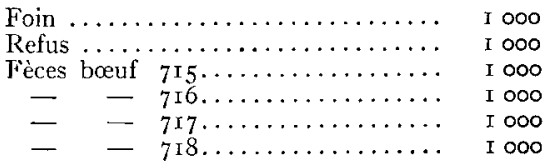

\begin{tabular}{|c|c|c|c|c|c|c|c|c|c|}
\hline Foin & & . & I 000 & 58,2 & $94 \mathrm{I}, 8$ & 24,8 & 55,2 & 395,9 & 341,8 \\
\hline Refus & & $\ldots \ldots \ldots \ldots \ldots \ldots \ldots \ldots$ & I 000 & 59,0 & $94 \mathrm{r}, \mathrm{O}$ & 20,2 & 32,6 & 489,4 & 298,9 \\
\hline èces & bœuf & $71_{5} \ldots \ldots \ldots \ldots \ldots$ & I 000 & I I 8,8 & $88 I, 2$ & 16,2 & 48,8 & $46 \mathrm{I}, 7$ & $27,3,3$ \\
\hline - & - & $716 \ldots \ldots \ldots \ldots \ldots$ & I 000 & 107,7 & 892,3 & $\mathrm{I} 5, \mathrm{I}$ & 47,0 & 485,7 & 269,2 \\
\hline - & - & $7 \times 7 \ldots \ldots \ldots \ldots \ldots$ & I 000 & 127,4 & 872,6 & I6,8 & $50, \mathrm{I}$ & 464,6 & 257,2 \\
\hline - & - & $7 \mp 8 \ldots \ldots \ldots \ldots$ & I 000 & 102,2 & 897,8 & 16,0 & 44,3 & 454,0 & 03,3 \\
\hline & & & & \multicolumn{6}{|c|}{ Période expérimentale } \\
\hline Foin & & & I. 000 & 44,2 & 955,8 & $22, \mathrm{I}$ & 38,6 & 429,0 & 355,7 \\
\hline Refus & & & 1000 & 70,8 & 929,2 & 25,7 & 30,6 & 400,2 & $344, \Gamma$ \\
\hline Pulpe & & $\ldots \ldots$ & I 000 & 105,0 & 895,0 & I6,I & 40,5 & 229,4 & $5 \mathbf{2 8 , 8}$ \\
\hline Fèces & bouf & $715 \ldots \ldots \ldots \ldots \ldots$ & I 000 & I 36,6 & 863,4 & 24,8 & $4 I, 5$ & $39 \mathrm{I}, 7$ & $28 \mathrm{r}, 4$ \\
\hline- & - & $716 \ldots \ldots \ldots \ldots \ldots$ & I 000 & I $33, \mathrm{I}$ & 866,9 & 22,9 & 40,9 & $4 I 2,0$ & 276,0 \\
\hline 一 & - & $717 \ldots \ldots \ldots \ldots \ldots$ & I 000 & $\mathrm{~T}_{4} \mathrm{I}, 2$ & 858,8 & 25,3 & $4 I, I$ & $3^{82,4}$ & 283,5 \\
\hline - & - & $\ldots \ldots \ldots \ldots \cdots$ & 1000 & I $3 x, 4$ & 868,6 & 22,8 & 43,6 & 416,0 & 272,0 \\
\hline
\end{tabular}

Période expérimentale

\begin{tabular}{|c|c|c|c|c|c|c|c|c|c|}
\hline Foin & & & I 000 & $5^{8,2}$ & $94 \mathrm{I}, 8$ & 24,8 & 55,2 & 395,9 & 341,8 \\
\hline Refus & & ... & I 000 & 59,0 & $94 \mathrm{I}, \mathrm{O}$ & 20,2 & 32,6 & 489,4 & 298,9 \\
\hline Fèces & bœuf & $7 I_{5} \ldots$ & I 000 & I I 8,8 & $88 \mathrm{I}, 2$ & 16,2 & 48,8 & $46 \mathrm{r}, 7$ & 273,3 \\
\hline - & - & $716 \ldots \ldots \ldots \ldots \ldots$ & I 000 & 107,7 & $89^{2}, 3$ & $\mathrm{I}, \mathrm{I}$ & 47,0 & 485,7 & 269,2 \\
\hline - & 一 & $7 \times 7 \ldots \ldots \ldots \ldots \ldots$ & I 000 & 127,4 & 872,6 & $\mathbf{1 6 , 8}$ & 50,1 & 464,6 & 257,2 \\
\hline - & - & $718 \ldots \ldots \ldots \ldots \ldots$ & I 000 & 102,2 & 897,8 & 16,0 & 44,3 & 454,0 & 303,3 \\
\hline & & & & \multicolumn{6}{|c|}{ Période expérimentale } \\
\hline Foin & & . & I. 000 & 44,2 & 955,8 & $22, \mathrm{I}$ & 38,6 & 429,0 & 355,7 \\
\hline Refus & & & 1000 & 70,8 & 929,2 & 25,7 & 30,6 & 400,2 & $344, \mathrm{I}$ \\
\hline Pulpe & & $\cdots$ & I 000 & I05,0 & $895, \circ$ & $x 6,1$ & 40,5 & 229,4 & $5 \mathbf{2 8 , 8}$ \\
\hline Fèces & oeuf & $\ldots \ldots$ & I 000 & I 36,6 & 863,4 & 24,8 & $4 I, 5$ & $39 \mathrm{I}, 7$ & $28 \mathrm{I}, 4$ \\
\hline- & - & $\ldots \ldots \ldots \ldots$ & I 000 & I33, I & 866,9 & 22,9 & 40,9 & 412,0 & 276,0 \\
\hline 一 & - & $717 \ldots \ldots \ldots \ldots \ldots$ & I 000 & $\mathrm{I}_{4} \mathrm{I}, 2$ & 858,8 & 25,3 & $4 \mathrm{I}, \mathrm{I}$ & $3^{82,4}$ & 283,5 \\
\hline - & - & $718 \ldots \ldots \ldots \ldots \ldots$ & I 000 & I $3 I, 4$ & 868,6 & 22,8 & 43,6 & 416,0 & 272,0 \\
\hline
\end{tabular}

(1) Les animaux n'ont pas laissé de refus en quantité suffisante pour effectuer une analyse complète.

$\begin{array}{rlllll}89,8 & 910, \mathrm{I} & 28, \mathbf{1} & 35,5 & \mathbf{2 7 6 , 4} & 432,4 \\ 40,9 & 959, \mathbf{I} & \mathbf{1 7 , 7} & 40,6 & \text { I } 87, \mathrm{I} & 624,9 \\ \text { I 26,9 } & 783, \mathrm{I} & 27,4 & 52,0 & 305,9 & 350,9 \\ \text { I 26,3 } & 873,7 & 25,8 & 52,6 & 307,4 & 358,9 \\ \text { I 23,2 } & 876,8 & 29,2 & 50,1 & 3 \mathbf{1 9 , 4} & 338,2\end{array}$

Brebis - 3 sujets

Période témoin

$87,0 \quad 9^{13}, 0 \quad 3^{2,4} \quad 3^{6, \mathrm{I}} \quad 3^{\mathrm{I} 8, \mathrm{I}} \quad 364,3$

$54,0 \quad 945,0 \quad$ I $5,5 \quad 24,8 \quad 509,0 \quad 3$ I 9,3

I $58,5 \quad 84 \mathrm{I}, 5 \quad 2 \mathrm{I}, 3 \quad 53,5 \quad 374,2 \quad 285,9$

I 64, I $\quad 835,9 \quad 23,0 \quad 55,5 \quad 332,3 \quad 309,8$

I $48,6 \quad 85 \mathrm{I}, 3 \quad 20, \mathrm{I} \quad 56,7 \quad 377,3 \quad 297,0$

Période expérimentale

$\begin{array}{rlllll}78,8 & 921,2 & 32,2 & 30, \mathrm{I} & 3 \mathrm{I} 2,9 & 384,9 \\ 55,4 & 944,6 & 16,3 & 23,7 & 479, \mathrm{I} & 344,2 \\ 69,5 & 930,5 & 17,4 & 36,9 & 2 \mathrm{IO}, 6 & 578,7 \\ \mathrm{I} 79,2 & 820,8 & 27,5 & 65,6 & 25 \mathrm{I}, 0 & 339,2 \\ \mathrm{I} 72,2 & 824,8 & 25,9 & 57,7 & 235,0 & 376,4 \\ \mathrm{I} 46,8 & 853,2 & 25, \mathrm{I} & 57,9 & 254,4 & 390, \mathrm{I}\end{array}$

Brebis - 3 sujets

$65, \mathrm{I} \quad 934,9 \quad \mathrm{I} 6, \mathrm{I} \quad 34,8 \quad 223,0 \quad 580,5$

$2 \mathrm{I} 2,0 \quad 788,0 \quad 34,5 \quad 55,7 \quad 213,8 \quad 3 \mathrm{II}, 7$

$202, \mathrm{I} \quad 797,9 \quad 32,5 \quad 65,2 \quad 225,7 \quad 3$ II,9

2 19,3 $\quad 780,7 \quad 35,0 \quad 63,6 \quad 19^{2,8} \quad 3^{\text {T } 4,6}$

Boufs - 4 sujets

Période témoin 
TABLEAU III (suite)

Période expérimentale

Groupe d'expérzences $n^{0} 6$ :

Foin $\ldots \ldots \ldots \ldots \ldots \ldots \ldots \ldots \ldots$ I 000

Refus bœuf noir .............. I 000

- $\quad-\operatorname{roux} \ldots \ldots \ldots \ldots \ldots \ldots$ I 000

Fèces bœuf noir............... I

$-\quad \rightarrow \quad r o u x \ldots \ldots \ldots \ldots \ldots$ I 000

Foin

1000

Refus bouf noir .............. I 000

Refus bouf roux .............. I ooo

Pulpe ................... r 000

Fèces bœuf noir................ I 000

$-\quad-\operatorname{roux} \ldots \ldots \ldots \ldots \ldots \ldots$ I 000

$\begin{array}{ll}\text { Mat. } & \text { Mat. } \\ \text { mine- } & \begin{array}{l}\text { orga- } \\ \text { rale }\end{array}\end{array}$

Azote grasse lose

Extractifs

non

Boufs - 2 sujcts

Période témoin

$\begin{array}{rlllll}73,9 & 926, \mathrm{I} & 24,0 & 44, \mathrm{I} & 401,3 & 396,3 \\ 56,5 & 943,5 & \mathrm{I} 8, \circ & 34,9 & 498,9 & 302, \mathrm{I} \\ 50,3 & 949,7 & \mathrm{I} 6,8 & 22,3 & 497,2 & 329,6 \\ \mathrm{1} 29,6 & 870,4 & 15,9 & 53, \mathrm{r} & 46,3,3 & 258,2 \\ \mathrm{I} 35,4 & 864,6 & \mathrm{I} 6,2 & 63,4 & 455,2 & 248,9\end{array}$

Période expérimentale

$\begin{array}{rlllll}76,7 & 923,3 & 28,4 & 37,3 & 360,9 & 355,0 \\ 65,4 & 934,6 & 20,4 & 35,3 & 448,6 & 328,5 \\ 57,8 & 942,2 & 20,1 & 33,7 & 460,1 & 328,1 \\ 70,5 & 929,5 & 18,0 & 30,4 & 223,4 & 567,9 \\ \text { I } 49,8 & 850,2 & 18,6 & 76,1 & 420,3 & 242,4 \\ \text { I } 52,5 & 847,5 & \text { I } 8,8 & 74,7 & 430,7 & 229,4\end{array}$

Les variances pour chaque animal, au cours d'une même périođe, se sont toujours montrées importantes. En fonction des aliments étudiés, nous avons constaté la prédominance, tantôt des influences individuelles, et tantôt des influences dépendant du régime distribué.

Le tableau IV ci-après', montre l'importance de cette variation dans les résultats d'observations.

TABLEAU IV

Teneur en matière sèches des matières fécales des brebis suivant les régimes et les animaux

\begin{tabular}{|c|c|c|c|c|}
\hline Régime & $\begin{array}{c}\text { Brebis } \\
\quad 11\end{array}$ & $\begin{array}{c}\text { Brebis } \\
12\end{array}$ & $\begin{array}{c}\text { Brebis } \\
13\end{array}$ & Moyenne \\
\hline Foin $+46 \%$ pulpe & 36,6 & 40,8 & 35,4 & 37,6 \\
\hline Pulpe ensilée $+5 \%$ balles & - & 43,7 & 55,5 & 49,6 \\
\hline Pulpe ensilée $+7 \%$ balles $\ldots \ldots \ldots$ & 46,7 & 48,5 & 38,6 & 44,5 \\
\hline Foin $\ldots \ldots \ldots \ldots \ldots \ldots \ldots \ldots \ldots \ldots \ldots \ldots$ & $4 \mathrm{~T}, \mathrm{~T}$ & 50,5 & 42,8 & 44,6 \\
\hline & $\begin{array}{c}\text { Brebis } \\
1\end{array}$ & $\begin{array}{c}\text { Brebis } \\
2\end{array}$ & $\begin{array}{c}\text { Brebis } \\
3\end{array}$ & Moyenne \\
\hline Foin & $\overline{32,0}$ & $\overline{35,8}$ & $\widetilde{38,2}$ & \\
\hline Foin $+34 \%$ pulpe & 38,4 & $\begin{array}{l}33,0 \\
43, \mathrm{I}\end{array}$ & 38,9 & $\begin{array}{l}35,3 \\
40,1\end{array}$ \\
\hline Pulpe $\ldots \ldots \ldots \ldots \ldots$ & 28 , I & 52,4 & 41,3 & 40,6 \\
\hline$\ldots \ldots \ldots \ldots \ldots$ & 33,0 & 43,6 & 39,6 & 38,7 \\
\hline Foin & 44,9 & 43,8 & $4 \mathrm{I}, \mathrm{O}$ & 43,2 \\
\hline Paille & 39,7 & 43,2 & 44,9 & 42,6 \\
\hline Marc de $p c$ & 56,9 & 55,2 & $57, \mathrm{I}$ & 56,4 \\
\hline
\end{tabular}

Chez les bœufs, les variations des teneurs en matières sèches des matières fécales se sont montrées très faibles. Ire tableau $V$ donne ces teneurs. Nous avons joint aux résultats des expériences ci-rapportées, ceux d'autres expériences effectuées sur ces mêmes animaux. 
TABLEAU V

Teneur en matière sèche des matières fécales des boufs suivant les régimes et les animaux

\begin{tabular}{|c|c|c|c|c|}
\hline Régime & $\begin{array}{c}\text { Beuuf } \\
715\end{array}$ & $\begin{array}{c}\text { Beut } \\
716\end{array}$ & $\begin{array}{c}\text { Bæuf } \\
717\end{array}$ & $\begin{array}{c}\text { Boouf } \\
718\end{array}$ \\
\hline 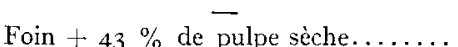 & $\overline{I 5.8}$ & $\overline{\mathrm{t}} \overline{6} .6$ & $\overline{17.1}$ & $\overrightarrow{180}$ \\
\hline Foin $\quad \ldots \ldots \ldots \ldots \ldots \ldots \ldots \ldots \ldots \ldots$ & 18,3 & $\mathrm{Ig}, 6$ & 17,3 & $\begin{array}{l}19,2 \\
19,2\end{array}$ \\
\hline Foin $+13 \%$ de pulpe sèche........ & $18, \mathrm{I}$ & 20,0 & 17,8 & 57,7 \\
\hline \multirow{2}{*}{ Pulpe ensilée + foin $\ldots \ldots \ldots \ldots \ldots$} & $\mathrm{I}_{5}, 8$ & I 7,7 & I 7,4 & 18,7 \\
\hline & $\begin{array}{l}\text { Bounf } \\
\text { noir } \\
-\end{array}$ & $\begin{array}{l}\text { Bouf } \\
\text { roux }\end{array}$ & & \\
\hline Foin & 18,7 & 17,3 & & \\
\hline Foin $+29 \%$ de pulpe se & $19, \mathrm{r}$ & 17,0 & & \\
\hline Foin $+50 \%$ de son $\ldots \ldots \ldots \ldots$ & 19,5 & $\mathbf{1 7 , 3}$ & & \\
\hline Foin $+25 \%$ de son $\ldots \ldots \ldots$. & 17,9 & 16,7 & & \\
\hline Foin $\quad \ldots \ldots \ldots \ldots \ldots \ldots$ & 19,0 & - & & \\
\hline Foin + pulpe sèche + son & 17,8 & I 5,8 & & \\
\hline
\end{tabular}

Le tableau VI donne les coefficients de digestibilité des composants des divers régimes témoins et expérimentaux, avec les résultats obtenus pour la pulpe, calculés par différence.

\section{Tableau VI}

Coefficients de digestibilités des divers régimes chez les ruminants

\begin{tabular}{|c|c|c|c|}
\hline \multirow{4}{*}{ 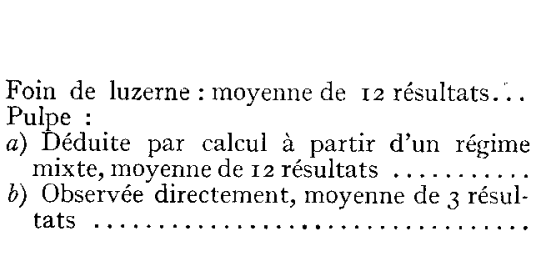 } & $\begin{array}{l}\text { Matière } \\
\text { sèche }\end{array}$ & $\begin{array}{l}\text { Matière } \\
\text { organique }\end{array}$ & Azote \\
\hline & $54,8 \bar{Y} \quad 0,8$ & ${ }_{57,4} \mp 0,9$ & $71,2 \bar{\mp}, 0,8$ \\
\hline & $73,0 \mp 4,9$ & $76,1 \stackrel{1}{\div} 4,4$ & $3^{6,2} \div 10,0$ \\
\hline & $70,7 \mp \quad 0,9$ & $76,9 \mp 0,6$ & $38,3 \mp \quad \mathrm{I}, 7$ \\
\hline & $\begin{array}{l}\text { Matières } \\
\text { grasses }\end{array}$ & $\begin{array}{l}\text { Matières } \\
\text { cellulosiques }\end{array}$ & $\begin{array}{l}\text { Extractifs } \\
\text { non azotés }\end{array}$ \\
\hline $\begin{array}{l}\text { Foin de luzerne : moyenne de I } 2 \text { résultats... } \\
\text { Pulpe de betterave : } \\
\text { a) Déduite par calcul à partir d'un régime }\end{array}$ & 46,0 工 I I,6 & $43,9 \mp \mathrm{r}, 3$ & $63,7 \mp 1,3$ \\
\hline $\begin{array}{l}\text { mixte, moyenne de is résult } \\
\text { b) Observée directement, moyen }\end{array}$ & $28,2 \div 14,2$ & So,o $\mp 7,5$ & $8_{4,0} \mp{ }_{4,8}$ \\
\hline & $45, \mathrm{I} \quad \mp^{\prime} \quad 2, \mathrm{I}$ & $73,2 \div \mathrm{I}, 7$ & $84,2=0,4$ \\
\hline
\end{tabular}

Nous constatons ainsi que la méthode par différence utilisée avec les données d'un nombre d'observations suffisamment grand, fournit des résultats pratiquement en bon accord avec ceux de la méthode directe.

Il faut mettre en évidence le parfait accord de nos résultats avec les plus récents travaux publiés en Suède, qui donnent comme coefficients de digestibilité de la pulpe mesurés avec des vaches laitières : 
La recherche des causes de variation observées d'une expérience à l'autre s'avère délicate car les différents facteurs à envisager ont été modifiés simultanément : qualité du foin, qualité de la pulpe, pourcentage de pulpe dans le régime mixte; l'insuffisance du nombre de données pour chacun de ces facteurs n'en permet pas une étude statistique.

La très forte dispersion des coefficients de digestibilité obtenus pour les matières grasses, s'explique par les difficultés particulières du dosage de ces substances dans les aliments comme dans les matières fécales ( ${ }^{1}$ ).

Le calcul de la valeur fourragère de la pulpe de composition moyenne effectué à l'aide des coefficients de digestibilité de l'expérience directe : matières azotées : 38 ; matières grasses : 45 ; matières cellulosiques : 73 ; extractifs non azotés : 84 donne le résultat suivant:

$$
\text { valeur fourragère par } \mathrm{kg}=0,804 \text {. }
$$

En nous basant sur les coefficients déterminés par différence : matières azotées : 36 ; matières grasses : 28 ; matières cellulosiques : 80 ; extractifs non azotés : 84 ; nous arrivons au résultat ci-après :

valeur fourragère par $\mathrm{kg}=0,807$.

qui peut être considérée comme pratiquement équivalente à la première.

\section{RESUME ET CONCLUSIONS}

I) La pulpe sèche de betteraves se classe parmi les aliments concentrés peu riches en matières azotées. Le séchage fournit une excellente solution du problème de la conservation de la pulpe qui est toujours délicat à réaliser par le procédé de l'ensilage, plus couramment utilisé en France. Mais les techniques du séchage exigent malheureusement un appareillage cô̂teux et une très forte dépense de combustible.

2) Nous avons procédé à l'étude de la digestibilité de la pulpe sèche sur les espèces suivantes: porcins; ovins, bovins.

3) La digestibilité de la pulpe sèche a été calculée par différence entre un régime témoin et un régime expérimental, différant du premier uniquement par la présence de pulpe.

4) Les coefficients de digestibilité de la pulpe sèche chez le porc sont pour chacun des composants :

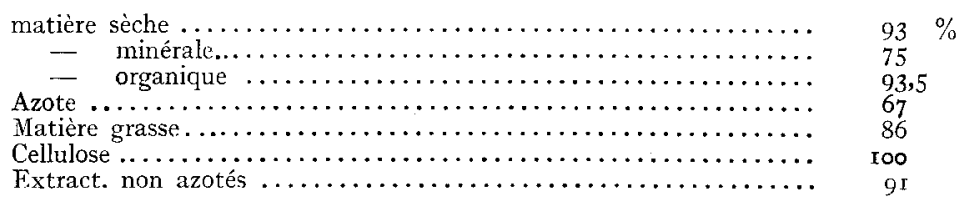

(1) Pour l'explication de ces faits. nous prions les lecteurs de vouloir bien se reporter au travail de MM. Françors et Leroy sur le dosage de ces matières grasses publié dans ce fascicule. 
5) La valeur fourragère correspondante est de $\mathrm{r}, \mathrm{I} 3 \mathrm{u}$. f. par $\mathrm{kg}$ si l'on considère la pulpe utilisée au cours de cette expérience et de $\mathrm{I}, 07 \mathrm{u}$. f. si l'on prend en considération la composition de la pulpe moyenne citée en référence au début de ce travail.

6) Les coefficients de digestibilité de la pulpe sèche, mesurés sur des bovins et des ovins soit directement, soit calculés par différence, sont entièrement comparables les uns aux autres et en accord avec les résultats publiés récemment en Suède.

\begin{tabular}{|c|c|c|c|}
\hline & $\begin{array}{c}\text { par } \\
\text { calcul } \\
-\end{array}$ & $\begin{array}{l}\text { par observation } \\
\text { directe }\end{array}$ & $\begin{array}{l}\text { résultats } \\
\text { Suédois }\end{array}$ \\
\hline Matière sèche $\ldots \ldots \ldots \ldots \ldots \ldots \ldots \ldots$ & $73,0 \%$ & $70,7 \%$ & \\
\hline 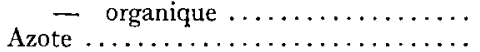 & 76,1 & 76,9 & 76,7 \\
\hline $\begin{array}{l}\text { Azote } \ldots \ldots \ldots \ldots \ldots \ldots \\
\text { Matières cellulosiques } \ldots \ldots \ldots \ldots \ldots \ldots\end{array}$ & $3^{6,2}$ & 38,3 & 43,7 \\
\hline $\begin{array}{l}\text { Matières cellulosiques } \ldots \ldots \ldots \ldots \ldots \ldots \\
\text { Extractifs non azotés } \ldots \ldots \ldots \ldots \ldots \ldots\end{array}$ & 80,0 & 73,2 & \\
\hline Extractifs non azotés ............. & 84,0 & 84,2 & \\
\hline
\end{tabular}

7) La valeur fourragère correspondante s'élève, selon les coefficients de digestibilité choisis à 0,804 et 0,807 , résultats qui peuvent être pratiquement confondus.

8) Limites de tolérance.

Nos observations expérimentales nous permettent d'affirmer qu'il existe un seuil maximum de tolérance pour la pulpe sèche, qui n'est pas le même pour les trois espèces animales que nous avons étudiées.

Pour les porcins, 1'apparition de troubles intestinaux se produit lorsque le pourcentage de pulpe atteint $20 \%$ de la ration, ce qui donne à penser que le seuil d'utilisation ne doit pas dépasser I $_{5} \%$.

Chez les bovins, les troubles n'apparaissent que pour un pourcentage supérieur à $45 \%$; le seuil d'utilisation se place donc aux environs de $35 \%$.

Pour les ovins qui paraissent présenter une tolérance plus grande que les bovins, ils convient également de limiter l'utilisation de la pulpe sèche, afin de ne pas déséquilibrer l'ensemble du régime. Nous pensons qu'il convient aussi de respecter cette même limite maximum de $35 \%$.

Ces indications correspondent au rapport existant entre la matière sèche de la pulpe et la matière sèche totale de la ration distribuée.

L'existence d'une limite de tolérance, qui est en relation avec la forte teneur des pulpes en matières pectiques, se trouve implicitement expliquée par les travaux de Michaux, sur l'utilisation digestive de ces substances (IO) (II). 


\section{TABLEAUX ANNEXES}

\section{Calcul des coefficients de digestibilité}

\begin{tabular}{|c|c|c|c|c|c|c|c|}
\hline Animal & $\begin{array}{l}\text { Matière } \\
\text { sèche }\end{array}$ & $\begin{array}{c}\text { Matière } \\
\text { minérale }\end{array}$ & $\begin{array}{c}\text { Matière } \\
\text { organique }\end{array}$ & Azote & $\begin{array}{c}\text { Matière } \\
\text { grasse }\end{array}$ & $\begin{array}{c}\text { Matières } \\
\text { cellulo- } \\
\text { siques }\end{array}$ & $\begin{array}{c}\text { Extractifs } \\
\text { non } \\
\text { azotés }\end{array}$ \\
\hline
\end{tabular}

Porc $n^{\circ} 1$

\begin{tabular}{|c|c|c|c|c|c|c|c|}
\hline Aliment ingéré .... & 2090,0 & г 39,8 & I 950,2 & $62, I$ & 85,7 & 299,9 & I 192,6 \\
\hline Fèces........... & 951,6 & 105,9 & 845,7 & I9,I & 35,2 & $3^{16,5}$ & 366 \\
\hline Retenus ....... & I 138,4 & 33,9 & I 104,5 & $4^{2,2}$ & 50,5 & - & \\
\hline Coefficient de digestibilité. & 54,5 & 24,2 & $5^{6,6}$ & 67,9 & 58,9 & o & \\
\hline \multicolumn{8}{|l|}{ Porc $n^{\circ} 2$} \\
\hline Aliment ingéré & I 980,0 & 132,5 & I 847,5 & $5^{8,8}$ & $8 \mathrm{r}, 2$ & $284, \mathrm{I}$ & I 12 \\
\hline Fèces............ & 911,6 & 91,6 & 820,2 & I9,I & $3^{1,2}$ & 301,1 & \\
\hline Retenus $\ldots \ldots \ldots$ & I 068,4 & 40,9 & 1027,3 & 39,7 & & & \\
\hline Coefficient de digestibilit & 53,9 & 30,9 & 55,6 & 67,5 & 61,6 & o & \\
\hline \multicolumn{8}{|l|}{ Pore $n^{0} 3$} \\
\hline Aliment ingéré & 2020,0 & $\mathrm{x} 35, \mathrm{I}$ & I 884,9 & 60,0 & 82,8 & 289,9 & \\
\hline Fèces........... & 941,6 & IOI,7 & 839,9 & 20,1 & 34,6 & 288,2 & \\
\hline Retenus & I 078,4 & 33,4 & I 045,0 & 39,9 & 48,2 & $\mathrm{I}, 7$ & \\
\hline Coefficient de digestib & 53,4 & 24,7 & 55,4 & 66,5 & 58,2 & 0,6 & \\
\hline
\end{tabular}

Expérience $n^{0} 1$. - Porcs. - Régime mixte $16 \%$ pulpe broyée Porc $n^{0} 1$

Aliment témoin .......... 2 I 31,8

\begin{tabular}{|c|c|}
\hline Pulpe & $\begin{array}{l}131,0 \\
448,2\end{array}$ \\
\hline Total ingéré. & 2580,0 \\
\hline Fèces... & $98 \mathrm{r}, 6$ \\
\hline Retenus. & I 598,4 \\
\hline Coefficient de digestibilité. & 61,9 \\
\hline Retenus pulpe. . & $\begin{array}{r}1101,0 \\
436,6\end{array}$ \\
\hline Coefficient de pulpe & \\
\hline
\end{tabular}

Porc no 2

Aliment témoin $\ldots \ldots \ldots \ldots \mid 2$ I 3 r,8

\begin{tabular}{|c|c|}
\hline Pulpe & 13,0 \\
\hline 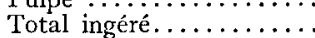 & $2 \begin{array}{r}440,2 \\
580,0\end{array}$ \\
\hline Fèces.............. & I $05 \mathrm{I}, 6$ \\
\hline Retenus ... & I 528,4 \\
\hline Coefficient de digestibilite & 59,2 \\
\hline Retenus témoin & I I 49,0 \\
\hline Retenus pulpe.. & 379,4 \\
\hline Coefficient & 84,6 \\
\hline
\end{tabular}

Pore $n^{\circ} 3$

Aliment témoin $\ldots \ldots \ldots \ldots \mid 2 \times 73,2$

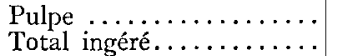

Total ingere............. 2630,0

Retenus ............ I 588,4

Coefficient de digestibilité.

Retenus témoin $\ldots \ldots \ldots$.....

Retenus pulpe............

60,4

I 160,5

427,9

Coefficient pulpe.

\begin{tabular}{r|r}
$\mathrm{I} 29,4$ & 2002,4 \\
27,2 & 421,0 \\
$\mathrm{I} 56,6$ & 2423,4 \\
$\mathrm{I} 05,4$ & 876,2 \\
$5 \mathrm{I}, 2$ & $\mathrm{I} 547,2$ \\
32,7 & 63,8 \\
$3 \mathrm{I}, 3$ & $\mathrm{I} \mathrm{I} 33,4$ \\
$\mathrm{I} 9,9$ & $4 \mathrm{I} 3,8$ \\
73,2 & 98,3
\end{tabular}

58,8

I 2,4

$7 \mathrm{I}, 2$

21,4

49,8

69,9

39,9

9,9

79,8

90,8
19,1
109,9
37,9
72,0
65,5
53,5
18,5
96,8

\begin{tabular}{r|r}
379,0 & I I 79,8 \\
79,7 & 248,0 \\
458,7 & I 427,8 \\
301,3 & 396,8 \\
I 57,4 & I 031,0 \\
34,3 & 72,2 \\
0 & 817,6 \\
I 57,4 & 213,4 \\
I97,5 & 86,3
\end{tabular}

129,4
27,2
156,6
97,1
59,5
38,0
40,0
19,5
71,6

2002,4

$42 \mathrm{I}, \mathrm{O}$

2423,4

954,5

I 468,9

60,6

I I I 3,3

355,6

84,5

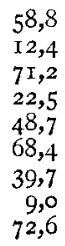

90,8

19,1
109,9

34,7

75,2

68,4

$\mathrm{I} 9,3$

I 9, I

IOO,O

\begin{tabular}{r|r}
379,0 & I $\mathrm{r} 79,8$ \\
79,7 & 248,0 \\
458,7 & I 427,8 \\
364,2 & $409, \mathrm{I}$ \\
94,5 & $\mathrm{I} 018,7$ \\
20,6 & $7 \mathrm{I}, 3$ \\
0 & 802,3 \\
94,5 & $2 \mathrm{I} 6,4$ \\
$\mathrm{I} 18,6$ & 87,2
\end{tabular}

2 O4I, 2 429 , I

2470,3 928,4

I $54 \mathrm{I}, 9$

62,4

I 130,8

$4 \mathrm{II}, \mathrm{I}$ 95,8

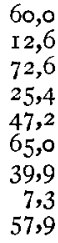

92,5
I 9,5
I 2,0
46,6
65,4
58,4
53,8
11,6
59,5

386,4

$8 \mathrm{I}, 2$ 467,6

318,2

$\mathrm{I} 49,4$

$3^{\mathrm{I}, 9}$

2,3

$\mathrm{I} 47$, I

I $8 \mathrm{I}, \mathrm{I}$ $\mathrm{r} 202,6$
252,8
$\mathrm{I} 455,4$
399,2
$\mathrm{I} 056,3$
72,6
801,0
255,3
101,0 


Animal $\left|\begin{array}{c}\text { Matière } \\ \text { sèche }\end{array}\right| \begin{gathered}\text { Matière } \\ \text { minérale }\end{gathered}\left|\begin{array}{c}\text { Matière } \\ \text { organique }\end{array}\right|$ Azote $\left\{\begin{array}{c}\text { Matière } \\ \text { grasse }\end{array}\left|\begin{array}{c}\text { Matières } \\ \text { cellulo- } \\ \text { siques }\end{array}\right| \begin{array}{c}\begin{array}{c}\text { Extractifs } \\ \text { non } \\ \text { azotés }\end{array} \\ \hline\end{array}\right.$

Expérience $n^{\circ}$ 1. - Porcs. - Régime mixte $16,8 \%$ pulpe éclatée

Porc $n^{\circ} 1$

\begin{tabular}{|c|c|}
\hline Aliment témoin & 285 \\
\hline Pulpe & 601,0 \\
\hline Total ingéré. & 3460,0 \\
\hline Fèces........ & I 311,6 \\
\hline Retenus ... & $2 \mathrm{I} 48,4$ \\
\hline Coefficient de digestibilité. & 62,1 \\
\hline Retenus témoin & I 558, I \\
\hline Retenus de la pulpe & 590,3 \\
\hline Coefficient de & \\
\hline
\end{tabular}

Porc no 2

Aliment témoin ......... 2809,4

\begin{tabular}{|c|c|}
\hline 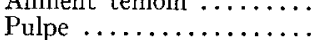 & $\begin{array}{l}809,4 \\
500,6\end{array}$ \\
\hline Total ingéré......... & 3400,0 \\
\hline Fèces............... & I 401,6 \\
\hline Retenus & I 998,4 \\
\hline Coefficient de digestibilité. & 58,8 \\
\hline Retenus témoin & I 5 I 4,3 \\
\hline Retenus de la pulpe. & $484, \mathrm{I}$ \\
\hline & \\
\hline
\end{tabular}

Porc $n^{\circ} 3$

Aliment témoin $\ldots \ldots \ldots \ldots \mid 2974,7$

\begin{tabular}{|c|c|}
\hline $\ln 2$ & (60 \\
\hline ingéré. & 360 \\
\hline 0 & I $38 x, 6$ \\
\hline & 2218 , \\
\hline st & \\
\hline & \\
\hline & \\
\hline
\end{tabular}

\begin{tabular}{r|r|}
$\mathrm{I} 87,2$ & $267 \mathrm{I}, 8$ \\
39,4 & 561,6 \\
226,6 & 3233,4 \\
$\mathrm{I} 43,7$ & $\mathrm{I} 167,9$ \\
82,9 & 2065,5 \\
36,6 & 63,9 \\
45,3 & $\mathrm{I} 5 \mathrm{I} 2,2$ \\
37,6 & 553,3 \\
95,4 & 98,5
\end{tabular}

80,1
I 6,8
96,9
30,5
66,4
68,5
54,4
$\mathrm{I} 2,0$
71,4

$\mathrm{I} r 9,5$
$25, \mathrm{I}$
$\mathrm{I} 44,6$
49,0
95,6
66,1
70,4
25,2
100,0

\begin{tabular}{c|r}
$50 x, 5$ & I $570, \mathrm{I}$ \\
I05,4 & $330, \mathrm{I}$ \\
606,9 & I 900,2 \\
409,0 & 515,5 \\
I97,9 & I 384,7 \\
32,6 & 72,9 \\
- & I $088, \mathrm{I}$ \\
I 97,9 & 296,6 \\
I87,8 & 89,8
\end{tabular}

\begin{tabular}{r|r}
$\mathrm{I} 84, \mathrm{O}$ & 2625,4 \\
38,7 & $55 \mathrm{I}, 9$ \\
222,7 & $3 \mathrm{I} 77,3$ \\
$\mathrm{I} 4 \mathrm{I}, 9$ & $\mathrm{I} 259,7$ \\
80,8 & $\mathrm{I} 9 \mathrm{I} 7,6$ \\
36,3 & 60,4 \\
56,9 & $\mathrm{I} 459,7$ \\
23,8 & 457,9 \\
$6 \mathrm{I}, 7$ & 83,0
\end{tabular}

78,7
16,5
95,2
33,0
62,2
65,3
53,1
9,1
55,2

II 7,4 24,7

$\mathrm{I} 42, \mathrm{I}$

$5,5,5$

60,6

60,9

$\mathrm{r} 4,3$

$5,9,2$

57,9

\begin{tabular}{r|r}
492,8 & I 540,2 \\
IO3,6 & 323,7 \\
596,4 & I 863,9 \\
437,7 & 556,5 \\
I 58,7 & I 307,4 \\
26,6 & 70,5 \\
- & I 047,3 \\
I 58,7 & $260, \mathrm{I}$ \\
I $53, \mathrm{I}$ & 80,4
\end{tabular}

I94,8
41,0
235,8
147,5
88,3
37,4
48,1
40,2
98,0

\begin{tabular}{|r|}
2779,8 \\
584,4 \\
3364,2 \\
$I 234, I$ \\
2 I $30, \mathrm{I}$ \\
63,3 \\
$\mathrm{I} 540,0$ \\
$590, \mathrm{I}$ \\
$10 \mathrm{I}, 0$
\end{tabular}

I 24,4
26, I
I 50,5
50,5
I00,0
66,4
72,4
27,6
I05,0

I 633,7

343,4

i 977 , I

537,3

I 439,8

73,0

I 088,0

351,8

102,3

Brebis $n^{0} 11$

\section{Expérience no 2. - Brebis. - Régime témoin}

Foin distribué ......... I 76I,7

Refus ...................................

Ingéré $\ldots \ldots \ldots \ldots \ldots \ldots \ldots \ldots$ I $47,{ }^{28,4}$

Fèces............................ 654,8

Retenus ........... 819,5

Coefficient de digestibilité. 55,5

\begin{tabular}{r|r|r} 
I $4 \mathrm{I}, 2$ & I 620,4 & 50,8 \\
$\mathrm{I} 5,3$ & 272,2 & 5,3 \\
$\mathrm{I} 25,9$ & $\mathrm{I} 348,2$ & 45,5 \\
80,5 & 574,3 & 12,2 \\
45,4 & 773,9 & 33,3 \\
$36, \mathrm{I}$ & 57,4 & 73,2
\end{tabular}

542,8

I 26,4

$6 \mathrm{I} 5,2$

$6{ }_{5}, 2$
$\times 95,2$

\begin{tabular}{l|r}
278,4 & 195,2 \\
138,0 & 420,0
\end{tabular}

Brebis $n^{0} 12$

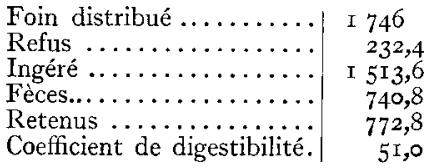

\begin{tabular}{|c|c|} 
I 40 & I 606 \\
I 2,3 & $220, \mathbf{I}$ \\
I 27,7 & I 385,9 \\
78,3 & 662,5 \\
49,4 & 723,4 \\
38,6 & 52,2
\end{tabular}

50,4

4,3
46,1

$\mathrm{r} 5,3$

30,8

66,3

68,2

Brebis no 13

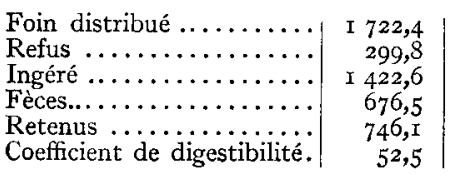

\begin{tabular}{|r|r|} 
I38,I & I 584,3 \\
I5,9 & 283,9 \\
I22,2 & I 300,4 \\
81,4 & 595,0 \\
40,8 & 705,4 \\
33,4 & 54,2
\end{tabular}

49,7
5,6
44,1
14,5
29,6
67,0

55,2
$\mathrm{I} 3,5$
$4 \mathrm{I}, 7$
29,8
$\mathrm{I} 1,9$
28,6




Animal $\left|\begin{array}{c}\text { Matière } \\ \text { sèche }\end{array}\right| \begin{gathered}\text { Matière } \\ \text { minérale }\end{gathered}\left|\begin{array}{c}\text { Matière } \\ \text { organique }\end{array}\right|$ Azote $\left|\begin{array}{c}\text { Matière } \\ \text { grasse }\end{array}\right| \begin{gathered}\text { Matières } \\ \text { cellulo- } \\ \text { siques }\end{gathered} \mid \begin{gathered}\text { Extractifs } \\ \text { non } \\ \text { azotés }\end{gathered}$

Expérience $n^{\circ} 2$. - - Brebis. - Régime mixte $46, \mathrm{x} \%$ pulpe

\begin{tabular}{|c|c|c|c|c|c|c|c|}
\hline Foin ingéré & 800,0 & 71,8 & 728,2 & 22,5 & 25,0 & $22 \mathrm{I}, \mathrm{I}$ & 345,9 \\
\hline Pulpe ingérée ......... & 708,0 & 29,0 & 679,0 & I $2, \mathrm{I}$ & 28,7 & I 32,4 & 442,4 \\
\hline Total ingéré. . . . . . . . & I 508,0 & 100,8 & I 407,2 & 34,6 & 53,7 & 353,5 & 700 \\
\hline 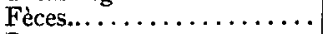 & 382,0 & 48,5 & 333,5 & Io, 5 & I9,9 & I 16,8 & I34, \\
\hline$\ldots \ldots \ldots \ldots$ & $\mathrm{I}$ I 26,0 & 52,3 & I 073,7 & $24, \mathrm{I}$ & 33,8 & $23^{6,7}$ & 654 \\
\hline Coefficient de digestibilité. & 75,0 & 51,8 & 76,3 & 69,5 & 63,0 & $67, I$ & 4 \\
\hline du foin $\ldots . .$. & 444,0 & 25,9 & 418,0 & $\mathrm{x} 6,5$ & 9,3 & 73,2 & 35 \\
\hline de la pulpe. & 682,0 & 26,4 & 655,7 & 7,6 & 24,5 & 163,5 & 418 \\
\hline ficient & 96,2 & 91,0 & 94,0 & 62,8 & 85,4 & I 23,0 & 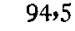 \\
\hline
\end{tabular}

\begin{tabular}{|c|c|c|c|c|c|c|c|}
\hline Foin ingéré & 860,0 & 77,2 & 782,7 & 24,2 & 30,6 & 229,0 & 372,0 \\
\hline Pulpe ingérée & 712,5 & 29,1 & 683,4 & I 2,2 & 28,9 & $\mathrm{r} 33,2$ & 445,2 \\
\hline Total ingéré..... & 572,5 & 106,3 & I $466, I$ & 36,4 & 59,5 & 262,2 & $8 \mathrm{I} 7,2$ \\
\hline 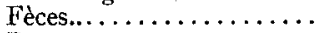 & 439,7 & 55,4 & $3^{84,2}$ & I I, 3 & 23,1 & I 35,2 & I 57,8 \\
\hline Retenus.......... & I I 32,8 & 50,9 & $x \circ 8 \mathrm{I}, 9$ & $25, \mathrm{I}$ & $3^{6,4}$ & 227,0 & 659,4 \\
\hline Coefficient de digestibilité. & 72,0 & 47,8 & 73,9 & 69,0 & $6 \mathrm{I}, 2$ & 62,8 & 80,7 \\
\hline Retenus du foin......... & 438,6 & 29,8 & 408,6 & 16,0 & I I, I & 89,3 & 208,7 \\
\hline Retenus de la pulpe. & 694,2 & $2 \mathrm{I}, \mathrm{I}$ & 673,3 & $9, \mathbf{I}$ & 25,3 & I 37,7 & 450,7 \\
\hline Coefficient de la pulpe... & 97,5 & 72,6 & 98,5 & 74,6 & 87,5 & I03,0 & IOI,O \\
\hline \multicolumn{8}{|l|}{ Brebis $n^{\mathbf{0}} 13$} \\
\hline Foin ingéré & 800,0 & 71,8 & 728,2 & 22,5 & 25,0 & $22 \mathrm{I}, \mathrm{I}$ & 345,9 \\
\hline Pulpe ingérée & 694,0 & 28,4 & 665,6 & II,9 & 28,2 & I 29,8 & 433,7 \\
\hline Total ingéré.... & I. 494,0 & 100,2 & I 393,8 & 34,4 & 53,2 & 350,9 & 779,6 \\
\hline Fèces......... & $404, \mathrm{I}$ & 49,8 & 354,3 & I 1,8 & 20,2 & $\mathrm{I} 29, \mathrm{I}$ & I 36,7 \\
\hline Retenus & I 089,9 & 50,4 & I 039,5 & 22,6 & 33,0 & 221,8 & 642,9 \\
\hline Coefficient de digestib & 73,0 & 50,1 & 74,7 & 65,6 & 62,1 & 61,9 & 82,9 \\
\hline Retenus du foin ... & 420,0 & 24,0 & 394,7 & I $5, \mathrm{I}$ & 7,2 & $97, \mathrm{I}$ & 197,9 \\
\hline Retenus de la pulpe. & 669,9 & 26,4 & 644,8 & 7,5 & 25,8 & I 24,7 & 445,0 \\
\hline Coefficient de la pulpe & 96,5 & 92,9 & 96,7 & 63,0 & $9 \mathbf{I}, 5$ & 96,0 & 103,0 \\
\hline
\end{tabular}

Expérience $n^{\circ} 3 .-$ Brebis. - Régime témoin

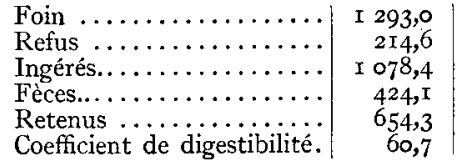

Brebis $n^{\circ} 2$

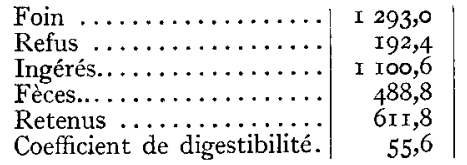

\begin{tabular}{r|} 
II 2,5 \\
IO,4 \\
I0 2,1 \\
80,2 \\
$2 \mathrm{I}, 9$ \\
$2 \pi, 4$
\end{tabular}

\begin{tabular}{r|}
$1 \mathrm{I} 80,5$ \\
182,0 \\
998,5 \\
408,6 \\
589,9 \\
59,1
\end{tabular}

42,0
3,0
39,0
$\mathrm{Ir}, 2$
27,8
$7 \mathrm{I}, 3$

46,5
4,8
$4 \mathrm{I}, 7$
$27, \mathrm{I}$
$\mathrm{I}, 6,6$
35,0

$4 \mathrm{II}, \mathrm{O}$ 97,9

$\mathrm{I} 62,4$

I 50,7

48 , I

\begin{tabular}{r|r|r|r|r|r}
$\mathrm{II} 2,5$ & $\mathrm{I} \mathrm{r} 80,5$ & 42,0 & 46,5 & $4 \mathrm{I} \mathrm{I}, \mathrm{O}$ & $47 \mathrm{I}, \mathrm{O}$ \\
7,0 & $\mathrm{I} 2 \mathrm{I}, 7$ & 2,0 & 3,2 & 65,5 & $4 \mathrm{I}, \mathrm{I}$ \\
$\mathrm{IO} 5,5$ & $\mathrm{I} 058,8$ & 40,0 & 43,3 & 345,5 & 429,9 \\
76,6 & 438,7 & $\mathrm{I0,4}$ & 29,2 & $\mathrm{I} 94,4$ & $\mathrm{I} 53,0$ \\
28,9 & $620, \mathrm{I}$ & 29,6 & $\mathrm{I} 4, \mathrm{I}$ & $\mathrm{I} 5 \mathrm{I}, \mathrm{I}$ & $\mathbf{2 7 6 , 9}$ \\
27,4 & 58,6 & 74,0 & 32,6 & 43,7 & 64,4
\end{tabular}

Brebis $n^{\circ} 11$

Brebis $n^{\circ} 12$

Brebis $n^{\circ} 1$

\section{Brebis $n^{\circ} 3$} Annales de Zootechnie. - $195^{2}$

.




\begin{tabular}{|c|c|c|c|c|c|c|c|}
\hline Animal & $\begin{array}{l}\text { Matière } \\
\text { sèche }\end{array}$ & $\begin{array}{c}\text { Matière } \\
\text { minérale }\end{array}$ & $\begin{array}{c}\text { Matière } \\
\text { organique }\end{array}$ & Azote & $\begin{array}{l}\text { Matière } \\
\text { grasse }\end{array}$ & $\begin{array}{l}\text { Matières } \\
\text { cellulo- } \\
\text { siques }\end{array}$ & $\begin{array}{c}\text { Extractifs } \\
\text { non } \\
\text { azotés }\end{array}$ \\
\hline
\end{tabular}

Expérience $n^{0}$ 3. - Brebis. - Régime mixte $33,6 \%$ pulpe Brebis $n^{0} 1$

Foin $\ldots \ldots \ldots \ldots \ldots \ldots$

Refus $\ldots \ldots \ldots \ldots \ldots \ldots \ldots \ldots$

Pulpe $\ldots \ldots \ldots \ldots \ldots \ldots \ldots$

Ingéré total ...........

Fèces.................

Retenus...............

Coefficient de digestibilité.

Retenus foin ...........

Retenus pulpe..........

Coefficient de la pulpe....

\section{Brebis $n^{\circ} 2$}

Foin

..........

Ingéré foin $\ldots \ldots \ldots \ldots \ldots$

Pulpe ................

Ingéré total $\ldots \ldots \ldots \ldots \ldots$

Feces..................

Retenus .............

Coefficient de digestibilité.

Retenus foin ...

Retenus pulpe............

Coefficient de la pulpe....

\begin{tabular}{r|r|r|}
$86_{3}, 0$ & 68,0 & 795,0 \\
224,9 & 12,5 & 212,4 \\
638,1 & 55,5 & 582,6 \\
349,4 & 24,3 & 325,1 \\
987,5 & 79,8 & 907,7 \\
365,9 & 65,6 & 300,3 \\
621,6 & 14,2 & 607,4 \\
62,9 & 17,8 & 66,9 \\
387,3 & 18,5 & 370,0 \\
234,3 & $-4,3$ & 237,4 \\
67,1 & $-17,7$ & 73,0
\end{tabular}

27,8
3,7
24,1
6,1
30,2
10,1
20,1
66,6
18,5
1,6
26,2

26,0
5,3
20,7
12,9
33,6
24,0
9,6
28,6
9,3
0,3
2,3

270,0

107,7

162,3

73,6

235,9

91,8

$\mathrm{r} 44, \mathrm{I}$

$6 \mathrm{I}, \mathrm{I}$

76,9

67,2

$9 \mathrm{I}, 3$

332,2

77,4

254,8

202,2

457,0

I 24, 1

332,9

72,8

I $78, \mathrm{I}$

I 44,8

7 I, 6

\begin{tabular}{r|r|r|r}
863 & 68,0 & 795 & 27,8 \\
$\mathrm{I} 68, \mathrm{I}$ & 9,3 & $\mathrm{I} 58,8$ & 2,7 \\
694,9 & 58,7 & 636,2 & $25, \mathrm{I}$ \\
$346, \mathrm{I}$ & $24, \mathrm{I}$ & 322,0 & 6,0 \\
$\mathrm{I} 04 \mathrm{I}, \mathrm{O}$ & 82,8 & 958,2 & $3 \mathrm{I}, \mathrm{I}$ \\
420,0 & 73,6 & 346,4 & 10,9 \\
$62 \mathrm{I}, 0$ & 9,2 & $6 \mathrm{I}, 8$ & 20,2 \\
59,7 & $\mathrm{I}, \mathrm{I}$ & 63,8 & 65,0 \\
386,4 & $\mathrm{I} 2,6$ & 376,0 & $\mathrm{I} 7,9$ \\
234,6 & $-3,4$ & 235,8 & 2,3 \\
67,8 & $-\mathrm{I} 4, \mathrm{I}$ & 73,2 & 38,3
\end{tabular}

26,0
4,0
22,0
12,8
34,8
24,2
10,6
30,5
7,7
2,9
22,6

270,0

80,5

I 89,5

72,9

262,4

98,7

I6 3,7

62,4

9 I, I

72,6

99,7

332,2

474,7

I 58 , I

$3 \mathrm{I} 6,6$

66,7

I 72,9

I 43,7

71,7

\section{Brebis $n^{0} 3$}

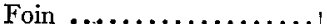

Refus $\ldots \ldots \ldots \ldots \ldots \ldots \ldots$

Ingéré foin............

Pulpe ..................

Ingéré total..$\ldots \ldots \ldots \ldots$

Fèces.................

Retenus ..............

Coefficient de digestibilité.

Retenus foin ...........

Retenus pulpe..........

Coefficient de la pulpe....

\begin{tabular}{r|r|r|r}
863,0 & 68,0 & 795,0 & 27,8 \\
126,6 & 7,0 & II 9,6 & 2,1 \\
736,4 & 61,0 & 675,4 & 25,7 \\
349,4 & 24,3 & $325, \mathrm{I}$ & $6, \mathrm{~T}$ \\
$\mathrm{I} 085,8$ & 85,3 & $\mathrm{I} 000,5$ & $3 \mathrm{I}, 8$ \\
445,3 & 65,4 & 379,9 & $1 \mathrm{I}, 2$ \\
640,5 & 19,9 & 620,6 & 20,6 \\
59,0 & 23,3 & 62,0 & 64,8 \\
410,2 & 16,7 & 396,0 & 19,0 \\
230,3 & 3,2 & 224,6 & 1,6 \\
65,9 & 13,2 & 69,1 & 26,2
\end{tabular}

$33^{2,2}$

43,6

288,6

202,2

490,8

I 73,7

$3 \mathrm{I} 7, \mathrm{r}$

64,6

I 85,9

\begin{tabular}{r|r}
78,2 & I 31,2 \\
106,2 & 64,9
\end{tabular}

Brebis $n^{\circ} 1$

Pulpe

$\ldots \ldots \ldots \ldots$

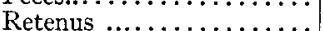

Coefficient de digestibilité.

Expérience no 4. - Brebis. - Pulpe pure

Brebis no 2

Pulpe

Fèces.......

Retenus $\ldots \ldots \ldots \ldots \ldots \ldots$

Coefficient de digestibilité.

Brebis no 3

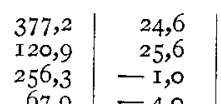

352,6

95,3

257,3

77,8

6,1
4,2
$I, 9$
$3 I, I$

6,1

$3 I, I$
84,1

25,8

58,3

69,3
219,0

37,7

$18 I, 3$
82,8

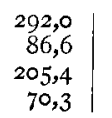

19,0
$\mathbf{1} 7,5$
1,5
7,9
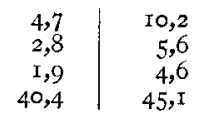

I 69,5

27,0

$\mathrm{I} 42,5$

$84, \mathrm{I}$

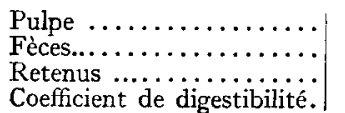

472,2

I 23, 3

348,9

73,9

\begin{tabular}{r|r|r}
30,7 & 441,5 & 7,6 \\
27,0 & 96,3 & 4,3 \\
3,7 & 345,2 & 3,3 \\
12,0 & 78,2 & 43,4
\end{tabular}

274, I

38,8

235,3 


Animal $\left|\begin{array}{c}\text { Matière } \\ \text { sèche }\end{array}\right| \begin{gathered}\text { Matière } \\ \text { minérale }\end{gathered}\left|\begin{array}{c}\text { Matière } \\ \text { organique }\end{array}\right|$ Azote $\left|\begin{array}{c}\text { Matière } \\ \text { grasse }\end{array}\right| \begin{gathered}\text { Matières } \begin{array}{c}\text { Extractifs } \\ \text { cellulo- } \\ \text { siques }\end{array} \\ \text { non } \\ \text { azotés }\end{gathered}$

Bouf $n^{\circ} 715$

Expérience $n^{\circ}$ 5. - Bœufs. - Régime témoin

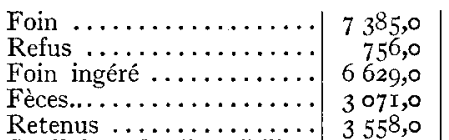

$\begin{array}{lr}\text { Retenus } \ldots \ldots \ldots \ldots \ldots \ldots & 55^{8,0} \\ \text { Coefficient de digestibilité. } & 53,7\end{array}$ Bouf $n^{\circ} 716$

\begin{tabular}{|c|c|c|c|c|c|}
\hline 429,8 & 6955,0 & I 83,3 & 407,7 & 2923,7 & $25^{24,2}$ \\
\hline 44,6 & $7 \mathrm{II}, \mathrm{O}$ & I 5,0 & 24,6 & 370,0 & 226,0 \\
\hline $3^{8} 5,2$ & 6244,0 & $\mathrm{I} 68,2$ & $3^{8} 3, \mathrm{I}$ & 2553,7 & 2298,2 \\
\hline 364,8 & 2706,0 & 49,9 & 149,9 & I 417,9 & 839,3 \\
\hline 20,4 & $35^{28,0}$ & I 18,3 & 233,2 & I $\mathbf{I} 35,8$ & . I 458,9 \\
\hline 5,3 & 56,7 & 70,4 & 60,9 & 44,5 & 63,5 \\
\hline
\end{tabular}

\begin{tabular}{r|r|}
423,6 & 6857,0 \\
48,4 & 773,0 \\
375,2 & 6084,0 \\
325,5 & 2697,0 \\
49,7 & 3387,0 \\
13,3 & 55,7
\end{tabular}

180,7

I6,4

I 64,3

45,5

I 18,8

72,3

401,9
26,8
$375, \mathrm{I}$
142,0
$233, \mathrm{I}$
$62, \mathrm{I}$

$\left|\begin{array}{r}2882,5 \\ 401,8 \\ 2480,7 \\ \text { I } 467,8 \\ \text { I } 012,9 \\ 40,8\end{array}\right|$

2488,6

245,4

2243,2

$8 \mathrm{I} 3,5$

I 429,7

63,7

\section{Bouf $n^{\circ} \% 1 \%$}

Foin ............. 7 906,o

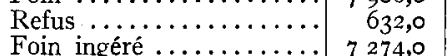

Fèces................ 3334,0

Retenus ............. 3940,0

Coefficient de digestibilité.

54,2

$460, \mathrm{r}$

37,3
422,8

7446,0

426,8

595,0
$685 \mathrm{I}, 0$

2909,0

$-2,0 \quad 3942,0$

196,2
$\mathrm{I} 2,6$
183,6
55,9
127,7
69,6

$43^{6,4}$

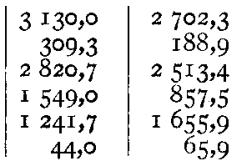

Bouf $n^{\circ} 718$

Foin $\ldots \ldots \ldots \ldots \ldots$

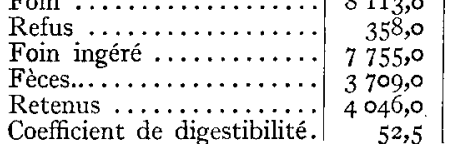

472,2

$45^{\mathrm{I}, \mathrm{I}}$

$764 \mathrm{r}, 0$
337,0
7304,0
3330,0
3974,0

201,3
7,2
194,1
59,4
134,7
69,4

447,8

\begin{tabular}{|c|c|}
\hline 3211,9 & 2773,0 \\
\hline 175,2 & 107,0 \\
\hline $3 \circ 3^{6,7}$ & 2666 , \\
\hline I 683 & I $\mathrm{I} 24,9$ \\
\hline I 352,8 & I $54 \mathrm{I}, \mathrm{I}$ \\
\hline 44 & \\
\hline
\end{tabular}

Expérience $n^{\circ} 5$. - Régime mixte $42 \%$ pulpe sèche

Bouf $n^{0} 715$

\begin{tabular}{|c|c|}
\hline Foin & 3836 \\
\hline Refus & $6 r, 0$ \\
\hline Foin ingéré & 3775,0 \\
\hline Pulpe & 2652,0 \\
\hline Total ingéré & $6427, \mathrm{o}$ \\
\hline Fèces............ & 2844,0 \\
\hline Retenus ......... & 3583,0 \\
\hline Coefficient de digestibilité. & 55,8 \\
\hline Retenus foin............ & 2027,2 \\
\hline $\begin{array}{l}\text { Retenus pulpe............ } \\
\text { Coefficient de la pulpe... }\end{array}$ & I 555,8 \\
\hline Loentient & \\
\hline
\end{tabular}

\begin{tabular}{r|r|r}
$I 69,6$ & 3666,4 & 84,7 \\
4,3 & 56,7 & 1,6 \\
$I 65,3$ & 3609,7 & 63,1 \\
278,5 & 2373,5 & 42,6 \\
443,8 & 5983,2 & $\mathrm{I} 25,7$ \\
388,5 & 2455,5 & 70,5 \\
55,3 & 3527,7 & 55,3 \\
12,5 & 59,0 & 43,9 \\
8,8 & 2046,7 & 58,5 \\
46,5 & $\mathrm{I} 48 \mathrm{I}, 0$ & $-3,3$ \\
16,7 & 62,4 & $-7,7$
\end{tabular}

I 48,I
I,9
I 46,2
107,1
253,3
118,0
135,3
53,4
89,0
46,3
43,2

I 645, I

I 364,5

21,0

I 620,7 I 343,5

608,4 I 402,4

$2229, \mathrm{I} \quad 2745,9$

I I I $4,0 \quad 800,3$

I I I 5, I I 945,6

$52,7 \quad 70,9$

$72 \mathrm{I}, 2 \quad 853, \mathrm{I}$

393,9 I 092,5

$64,8 \quad 77,9$

\title{
Beuf $n^{\circ} 716$
}

Foin ............... 3313,0

Refus $\ldots \ldots \ldots \ldots \ldots \ldots$

Pulpe $\ldots \ldots \ldots \ldots \ldots \ldots \ldots$

Total ingéré............

Fèces................

Coefficient de digestibilité.

3313,0
46,0
3267,0
2888,0
6 I 55,0
2541,0
3614,0
58,7
I 738,0
I 876,0
64,9

146,4

I 33,

73,2

I 27,9

1,4
I 26,5
I 6,7
243,2
I03,9
I 39,3
57,3
78,6
40,7

\begin{tabular}{|r|r} 
I 421,3 & I 178,4 \\
I 8,4 & I 5,8 \\
I 402,9 & I I 62,6 \\
662,5 & I 527,2 \\
2065,4 & 2689,8 \\
I 046,9 & 702,8 \\
I 018,5 & I 987,0 \\
49,3 & 73,9 \\
572,4 & 740,6 \\
446,1 & I 246,4 \\
67,3 & 81,6
\end{tabular}

$3 \circ 3,2$

446,3

338,2

108,1

24,2
19,0

89,1

Retenus pulpe...........

64,9

29,4

$3+23,9$

2584,8

$57 \circ 9,7$

2202,8

3506,9

$6 r, 4$

I 740,0

68,4

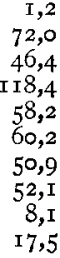

34,9

\begin{abstract}
67,3
\end{abstract}




\begin{tabular}{|c|c|c|c|c|c|c|c|}
\hline Animal & $\begin{array}{l}\text { Matière } \\
\text { sèche }\end{array}$ & $\begin{array}{l}\text { Matière } \\
\text { minérale }\end{array}$ & $\begin{array}{c}\text { Matière } \\
\text { organique }\end{array}$ & Azote & $\begin{array}{c}\text { Matière } \\
\text { grasse }\end{array}$ & $\begin{array}{l}\text { Matières } \\
\text { cellulo- } \\
\text { siques }\end{array}$ & $\begin{array}{c}\text { Extractifs } \\
\text { non } \\
\text { azotés }\end{array}$ \\
\hline
\end{tabular}

Expérience $n^{\circ}$ 5. - Régime mixte $4^{2} \%$ pulpe sèche (suite)

Bouf $n^{0} 717$

\begin{tabular}{|c|c|c|c|c|c|c|c|}
\hline Foin & 28360 & 606 & 666 & 847 & $88^{1}$ & $6 a=6$ & 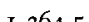 \\
\hline Refus & 0,0 & 0,6 & $\begin{array}{r}3 \\
8,4\end{array}$ & $\begin{array}{r}04,1 \\
0,2\end{array}$ & 0,3 & $\begin{array}{r}+43,6 \\
3,6\end{array}$ & $\begin{array}{r}3,1 \\
3,1 \\
304,5\end{array}$ \\
\hline Foin ingéré $\ldots . . \ldots \ldots$ & 3827,0 & I 69,0 & 3658,0 & 84,5 & $I_{47,8}$ & I 642,0 & I 361,4 \\
\hline Pulpe .......... & 2740,0 & 287,7 & 2452,8 & 44,0 & I10,7 & 628,6 & I 448,9 \\
\hline Total ingéré........ & 6567,0 & 456,7 & $6 \pm 10,3$ & I 28,5 & 258,5 & 2270,6 & $28 x 0,3$ \\
\hline Fèces............ & 3 I I 2,0 & 439,4 & 2672,6 & 78,7 & I 27,9 & I I 90,0 & $88 z, 3$ \\
\hline Retenus & 3455,0 & II 7,3 & 3437,7 & 49,8 & I 30,6 & I 080,6 & 1928,0 \\
\hline Coefficient de digestibilité. & 52,6 & 25,7 & 56,3 & 38,7 & $5 \circ, 5$ & 47,6 & 68,6 \\
\hline Retenus foin ........... & 2074,2 & - & 2107,3 & 58,8 & 28,4 & 722,5 & 897,2 \\
\hline Retenus pulpe........ & I 380,8 & I I 7,3 & I 330,7 & - 9,0 & 39,5 & $358, \mathrm{I}$ & I 030,8 \\
\hline Coefficient & 50,4 & $4 \mathrm{I}, \mathrm{I}$ & 54,4 & $-20,4$ & 35,7 & 56,9 & $7 \mathrm{I}, 2$ \\
\hline \multicolumn{8}{|l|}{ Bout no 718} \\
\hline Foin & 4097,0 & $\mathrm{I} 8 \mathrm{I}, \mathrm{I}$ & $39 \mathbf{5}, 9$ & $9 \circ, 5$ & I $58, I$ & I 757,6 & I 457,3 \\
\hline Refus & $\mathrm{I} 2, \mathrm{O}$ & 0,8 & I I, 2 & 0,3 & 0,4 & 4,8 & $4, \mathrm{I}$ \\
\hline Foin ingéré & 4085,0 & 180,3 & 3904,7 & 90,2 & I 57,7 & I 752,8 & I 453,2 \\
\hline Pulpe .... & 2904,0 & 304,9 & 2599,3 & 46,7 & I I 7,3 & 666,2 & I $53^{6,6}$ \\
\hline Total ingé & 6989,0 & 485,2 & 65 & I 36,9 & $275, \mathrm{o}$ & 2419,0 & 2989,8 \\
\hline es... . . . & $33^{29}, \mathrm{a}$ & 437,4 & $289 \mathrm{I}, 6$ & 76,0 & $\mathrm{I} 45, \mathrm{I}$ & I 384,9 & 905,5 \\
\hline & 3660,0 & 47,8 & 3612,4 & 60,9 & I 29,9 & I $034, \mathrm{I}$ & 2084,3 \\
\hline Coefficient de digestibilité. & $5^{2,4}$ & & 5,5 & 60,8 & 47,2 & 42,8 & 69,7 \\
\hline$\ldots \ldots \ldots$ & 2132,4 & 28,8 & 2 I 2 & 62,6 & 98,2 & $78 \mathrm{I}, 7$ & 839,9 \\
\hline Retenus pulpe $\ldots \ldots \ldots \ldots$ & I 527,6 & I 9,0 & I 488,2 & $-1,7$ & $3 \mathrm{I}, 7$ & 252,4 & I 243,4 \\
\hline Coefficient de la pulpe... & $5^{2,7}$ & 62,0 & 57,2 & $-3,6$ & 26,9 & $3^{8,0}$ & $8 \mathrm{I}, \mathrm{O}$ \\
\hline
\end{tabular}

\section{Expérience $n^{\circ}$ 6. - Boufs. - Période témoin}

Foin distribué .......... I0 200,0

Foin refusé ............ 967,0

Foin ingéré ............ 9233,0

Fèces.......................... 3 80

Retenus ............ 5432 ,

Coefficient de digestibilité. $\quad 58,8$

\section{Boeuf roux}

Foin distribué .......... Io 200,0

Foin refusé .......... I 5 II,O

Foin ingéré .......... 8689,0

Fèces................. 39 I 7,0

Retenus ............. 47772,2

Coefficient de digestibilité. $\quad 54,9$

\begin{tabular}{r|r|r}
$753, \mathrm{I}$ & 9446,2 & 244,8 \\
54,6 & $9 \mathrm{I} 2,4$ & 17,4 \\
699,2 & 8533,8 & 227,4 \\
492,6 & 3308,4 & 60,4 \\
206,6 & 5225,4 & 167,0 \\
29,5 & $6 \mathrm{I}, 2$ & 73,4
\end{tabular}

\begin{tabular}{r|r|r}
753,8 & 9446,2 & 244,8 \\
76,0 & I 435,0 & 25,4 \\
677,8 & 8 OI I,2 & 219,4 \\
530,4 & 3386,6 & 63,5 \\
147,4 & 4624,6 & 155,9
\end{tabular}

\begin{tabular}{r|r|r}
449,8 & 4093,3 & 3430,3 \\
33,7 & 482,4 & $392, \mathrm{I}$ \\
$4 \mathrm{I} 6, \mathrm{I}$ & $36 \mathrm{IO}, 9$ & $3 \mathrm{I} 38,2$ \\
$20 \mathrm{I}, 9$ & $\mathrm{I} 76 \mathrm{I}, 2$ & $98 \mathrm{I}, 6$ \\
$2 \mathrm{I} 4,2$ & $\mathrm{I} 849,7$ & $2 \mathrm{I} 56,6$ \\
$5 \mathrm{I}, 5$ & $5 \mathrm{I}, 2$ & 68,7
\end{tabular}

\begin{tabular}{r|r|r}
449,8 & 4093,3 & 3430,3 \\
33,7 & $75 \mathrm{I}, 3$ & 498,0 \\
$4 \mathrm{I} 6, \mathrm{I}$ & 3342,0 & 2932,3 \\
248,3 & $\mathrm{I} 783,0$ & 974,9 \\
$\mathrm{I} 67,8$ & $\mathrm{I} 559,0$ & $\mathrm{I} 957,4$ \\
40,3 & 46,6 & 66,8
\end{tabular}

Expérience no 6. - Bœufs. - Régime mixte $29,5 \%$ pulpe Bout noir

\begin{tabular}{|c|c|c|c|c|c|c|c|}
\hline Foin ... & 6867,0 & $5^{26,7}$ & 6340,3 & 195,0 & $25^{6, x}$ & 2478,3 & 2437,8 \\
\hline Refus ............... & $454, \circ$ & 29,7 & 424,3 & 9,3 & 16,9 & 203,7 & I 49, I \\
\hline Foin ingéré ............ & $64 \mathrm{I} 3,0$ & 497,0 & 5916,0 & 185,7 & $40, I$ & 274,6 & 2288,7 \\
\hline Pulpe $\ldots \ldots \ldots \ldots$ & 2575 , o & I 81,5 & 2393,5 & 46,3 & 78,3 & 575,2 & I 462,3 \\
\hline Total ingéré. & $8988, \circ$ & 678,5 & 8309,5 & 232,0 & 318,4 & 2849,8 & $375 \mathrm{I}, \mathrm{O}$ \\
\hline 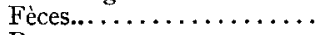 & $33^{1} 3,0$ & 496,3 & $28 \mathrm{I} 6,7$ & 61,6 & 252,1 & I 392,4 & 803,1 \\
\hline Retenus & 5675,0 & $I 82,2$ & 5492,8 & 170,4 & 66,3 & I 457,4 & 2947,9 \\
\hline Coefficient de digestibilité. & $63, \mathbf{I}$ & 26,8 & 66,1 & 73,4 & 20,8 & $5^{I, I}$ & 78,5 \\
\hline Retenus foin........... & 3770,8 & 146,6 & 3620,6 & I $3^{6,3}$ & 123,6 & I I 64,6 & I 572,3 \\
\hline Retenus pulpe......... & I 904,2 & 35,6 & I 872,2 & $34, \mathrm{I}$ & $-57,3$ & 282,8 & I 375,6 \\
\hline Coefficient de la pulpe. & $74, \mathrm{I}$ & 19,6 & 78,2 & 73,4 & $-72,3$ & 50,9 & 94,2 \\
\hline
\end{tabular}




\begin{tabular}{|c|c|c|c|c|c|c|c|}
\hline Animal & $\begin{array}{l}\text { Matière } \\
\text { sèche }\end{array}$ & $\begin{array}{c}\text { Matière } \\
\text { minérale }\end{array}$ & $\begin{array}{c}\text { Matière } \\
\text { organique }\end{array}$ & Azote & $\begin{array}{c}\text { Matière } \\
\text { grasse! }\end{array}$ & $\begin{array}{l}\text { Matières } \\
\text { cellulo- } \\
\text { siques }\end{array}$ & $\begin{array}{l}\text { Extractifs } \\
\text { non } \\
\text { azotés }\end{array}$ \\
\hline
\end{tabular}

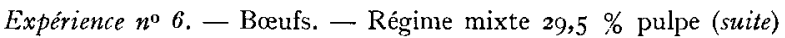
Bauf roux:

\begin{tabular}{|c|c|c|c|c|c|c|c|}
\hline Foin ......... & 6867,0 & 527,7 & 6340,3 & I 95,0 & $256, \mathrm{I}$ & 2472,3 & 2437,8 \\
\hline Refus ........... & 719,0 & 41,6 & 677,4 & $\mathrm{I} 4,4$ & 24,2 & 330,8 & 235,9 \\
\hline Foin ingéré $\ldots . . \ldots$ & $6 \mathrm{I}_{4} 8,0$ & $485, I$ & 5662,9 & I 80,6 & 231,9 & $2 \mathrm{I} 47,5$ & 2201,9 \\
\hline Pulpe .......... & 2548,0 & I 79,6 & 2368,4 & 45,8 & 77,5 & 569,2 & I 447,0 \\
\hline Total ingéré. & 8696,0 & 664,7 & 8031,3 & 226,4 & 309,4 & 2716,7 & 3648,9 \\
\hline Fèces............ & $3 \pm 62,0$ & 482,2 & 2680,0 & 59,4 & $23^{6,2}$ & r $36 \mathrm{r,9}$ & 725,4 \\
\hline Retenus $\ldots \ldots \ldots \ldots \ldots \ldots$ & $5534, \circ$ & $\mathrm{I} 82,5$ & $535^{r}, 3$ & 167,0 & 63,2 & I 354,8 & 2923,5 \\
\hline Coefficient de digestibilité. & 63,6 & 27,4 & 66,6 & 73,7 & 20,4 & 49,9 & 80,1 \\
\hline Retenus foin...... & 3375,0 & 105,3 & 3267,5 & 128,4 & 93,5 & I 000,7 & I 470,9 \\
\hline Retenus pulpe....... & 2 I 59,0 & 77,2 & 2083,8 & 38,6 & $-30,3$ & $354, \mathrm{I}$ & I 452,6 \\
\hline Coefficient de la pulpe.... & 84,7 & 43,0 & 88,0 & 84,3 & $-39,0$ & 62,2 & 100,0 \\
\hline
\end{tabular}

\section{BIBLIOGRAPHIE -- RÉFÉRENCES}

(1) Journal Officiel, Paris, 5 avril 1948.

(2) Leroy (A. M.). Normes pour l'alimentation énergétique. Rapports généraux du $5^{\mathrm{e}}$ congrès international de zootechnie. Paris 1949, p. 7 .

(3) Leroy (A.-M.), Michaux (A.). François (A.), Notes de laboratoire sur le dosage des matières cellulosiques par la méthode Weende. Congrès international des Industries Agricoles, Paris, 1948.

(4) SAYloR (Ch.-F.). - Sugar beet pulp as animal food. Progress of Beet : Sugar Industry I 902 .

(5) Pfeiffer (Th.). - Die Verdaulichkeit getrockneter Rübenschnitzel sowie die Bestimmung der Verdauungs coefficienten stickstoffhaltiger Futterbestandtheile im Algemeinen. J. F. Landzirts, 34, p. 425, 1886.

(6) Fingerling (G.). - Einfluss fettreicher und fettarmer Kraftfuttermittel auf die milchsecretion bei verschiedenem grundfutter. Landw. Versuchst, 64, p. 299, Igo6.

(7) Folke JARI. - Utfodringsförsök med ensilerad och terkad betmassa samt sulfitjästtill mjolkkor. Lantbrukshögskolan. Husdjursförsöksanstalten. Meddelande n. r., 31, p. I, I 938 .

(8) Leroy (A.-M.), LÉRy (G.). - Utilisation des déchets de corne hydrolysés pour l'alimentation du porc. Annales Agronomiques, 2, p. 264, I947.

(9) Leroy (A.-M.), Lucas (J.). - Les équivalents fourragers.

(IO) Leroy (A.-M.), MichauX (A.). - L'utilisation des matières pectiques par l'organisme animal. C. R. Acad. Sci. Paris, 229, p. ro34, 1950.

(i ) Michaux (A.). - - Les substances réductrices d'origine pectique au cours de la digestion chez les ruminants. C. R. Acad. Sci. Paris, 230, p. 2051, 1950. 